Received: 21 March 2017

Accepted: 4 July 2017

Published online: 25 July 2017

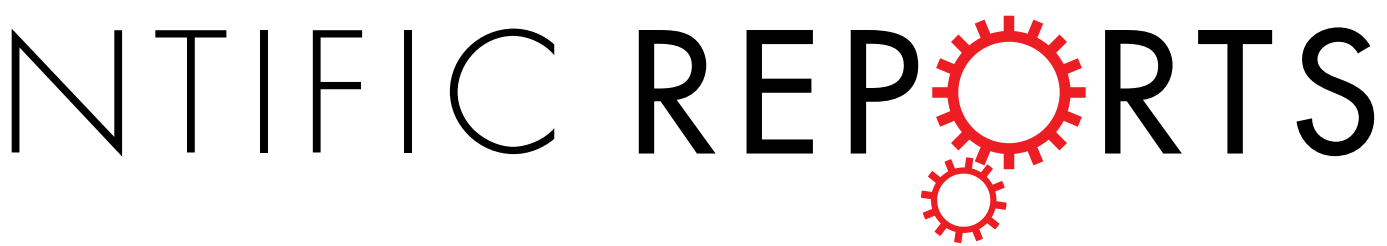

\title{
Virus and dsRNA-triggered
} transcriptional responses reveal key components of honey bee antiviral defense

\begin{abstract}
Laura M. Brutscher ${ }^{1,2,3}$, Katie F. Daughenbaugh ${ }^{1,3}$ \& Michelle L. Flenniken $\mathbb{1}^{1,2,3}$
Recent high annual losses of honey bee colonies are associated with many factors, including RNA virus infections. Honey bee antiviral responses include RNA interference and immune pathway activation, but their relative roles in antiviral defense are not well understood. To better characterize the mechanism(s) of honey bee antiviral defense, bees were infected with a model virus in the presence or absence of dsRNA, a virus associated molecular pattern. Regardless of sequence specificity, dsRNA reduced virus abundance. We utilized next generation sequencing to examine transcriptional responses triggered by virus and dsRNA at three time-points post-infection. Hundreds of genes exhibited differential expression in response to co-treatment of dsRNA and virus. Virus-infected bees had greater expression of genes involved in RNAi, Toll, Imd, and JAK-STAT pathways, but the majority of differentially expressed genes are not well characterized. To confirm the virus limiting role of two genes, including the well-characterized gene, dicer, and a probable uncharacterized cyclin dependent kinase in honey bees, we utilized RNAi to reduce their expression in vivo and determined that virus abundance increased, supporting their involvement in antiviral defense. Together, these results further our understanding of honey bee antiviral defense, particularly the role of a non-sequence specific dsRNAmediated antiviral pathway.
\end{abstract}

Globally, honey bees (Apis mellifera) and other insects are important pollinators of plants in both natural and agricultural landscapes. Insect pollination services are valued worldwide at $\$ 175$ billion annually ${ }^{1}$, and in the United States honey bee pollination is valued at $\$ 14.6$ billion annually ${ }^{2}$. Commercially managed honey bee colonies, which are the primary pollinators of numerous agricultural crops, have experienced high annual mortality in the U.S. (i.e., 33\% average annual loss since 2006) and parts of Europe ${ }^{3-5}$. Multiple abiotic and biotic factors, including pathogens, contribute to colony losses ${ }^{6,7}$. Pathogen incidence and abundance have been positively correlated with Colony Collapse Disorder (CCD)-affected colonies in the U.S. ${ }^{3,6,8}$ and colony losses in different regions of North America, South America, and Europe , $^{7-17}$. Honey bees are eusocial insects that live in colonies comprising approximately 40,000 sterile female workers, hundreds of male bees, and a single reproductive queen bee. Honey bees are often infected with pathogens including viruses, fungi, bacteria, and trypanosomatids, and they are typically parasitized by the Varroa destructor mite (reviewed in ref. 18).

The largest group of honey bee infecting pathogens are positive sense single-stranded viruses, including several Dicistroviruses (e.g., Israeli acute paralysis virus, Kashmir bee virus, Acute bee paralysis virus, and Black queen cell virus), Iflaviruses (e.g., Deformed wing virus, Sacbrood virus, and Slow bee paralysis virus), as well as taxonomically unclassified viruses (e.g., Chronic bee paralysis virus and the Lake Sinai virus group (reviewed in refs 19 and 20)). Honey bee-associated viruses exhibit variable pathogenicity and may cause deformity, paralysis, death, or remain asymptomatic (reviewed in refs 19 and 20). However, asymptomatic infections are commonly reported at levels of over $10^{7}$ virus equivalents (i.e., genomes and transcripts) per bee ${ }^{21}$, thus they likely affect bee physiology and health.

${ }^{1}$ Department of Plant Sciences and Plant Pathology, Montana State University, Bozeman, MT, USA. ${ }^{2}$ Department of Microbiology and Immunology, Montana State University, Bozeman, MT, USA. ${ }^{3}$ Pollinator Health Center, Montana State University, Bozeman, MT, USA. Correspondence and requests for materials should be addressed to M.L.F. (email: michelle.flenniken@montana.edu) 


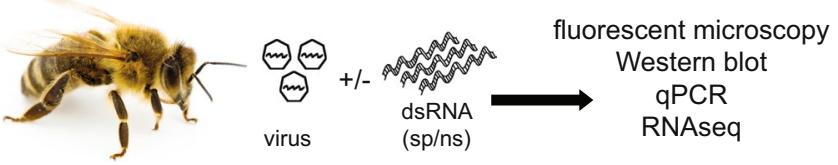

$6,48,72 \mathrm{hpi}$

Figure 1. Schematic of honey bee virus infection trials. Schematic representation of the experiments performed to investigate honey bee antiviral immune mechanisms, including dsRNA triggered immune responses. Honey bees were infected with virus, SINV-GFP, and/or treated with dsRNA, which was composed of either virusspecific dsRNA (sp-dsRNA) or nonspecific dsRNA (ns-dsRNA). Bees were collected at 6, 48, and 72 hours post infection (hpi); virus abundance in individual bees was assessed by fluorescence microscopy, Western blot analysis, and qPCR. Honey bee gene expression was assessed using RNASeq. Honey bee image courtesy of Kathy Keatley Garvey; used with permission.

Like other insects, honey bee antiviral responses include autophagy, apoptosis, eicosanoid biosynthesis, endocytosis, melanization, the JAK/STAT (Janus Kinase/Signal Transducer and Activator of Transcription), Toll, NF- $\kappa B$ (Nuclear Factor $\kappa B$ ), JNK (c-Jun N-terminal kinase), and MAPK (Mitogen-Activated Protein Kinases) pathways, and RNA interference (RNAi) ${ }^{9,15,22-26}$. RNAi is a post-transcriptional, sequence-specific, gene silencing mechanism and the small interfering RNA (siRNA)-mediated pathway is one of the primary insect antiviral defense mechanisms ${ }^{27-37}$. Correspondingly, several studies have shown that administration of virus-specific dsRNA or siRNA reduced viral load in honey bees ${ }^{36,38-40}$. Furthermore, CCD-affected colonies had higher amounts of virus-specific $22 \mathrm{nt}$ siRNAs as compared to non-CCD-affected colonies ${ }^{37}$ and early field studies suggested that honey bees fed IAPV-specific dsRNA had increased honey production and larger colony size ${ }^{41}$.

While experimental introduction of virus-specific dsRNA reduced honey bee virus infections, likely via $\mathrm{RNAi}^{36,38-40}$, non-sequence-specific dsRNA (ns-dsRNA) has also been shown to reduce virus abundance and affect gene expression in honey bees and bumble bees ${ }^{22,42-45}$. The more prominent role of ns-dsRNA mediated reduction in virus abundance in eusocial hymenopteran insects (e.g., honey bees and bumble bees), as compared to solitary insects (e.g., fruit flies and mosquitos) that do not exhibit this response, may reflect an evolutionary adaptation to limit virus transmission within colonies using general non-virus specific antiviral responses. In mammals, dsRNA serves as a virus-associated molecular pattern (VAMP) that is recognized by pathogen recognition receptors (PRRs), such as Toll-like receptor 3 (TLR3), Protein kinase R (PKR), Retinoic acid-inducible gene 1 (RIG-I), and Melanoma differentiation-associated gene 5 (MDA-5), and results in induction of the antiviral interferon response ${ }^{46}$. Similarly, Dicer, which is the endoribonuclease involved in RNAi, also serves as a dsRNA sensor that induces expression of antiviral defense genes (e.g., vago) in fruit flies, mosquitoes, and bumble bees ${ }^{44}$, 47-49. However, the role of specific genes in honey bee antiviral defense, particularly nonspecific dsRNA-mediated antiviral responses, are not well characterized.

Previously, we determined that treating honey bees with either virus sequence-specific-dsRNA (sp-dsRNA), or non-sequence specific dsRNA (ns-dsRNA), decreased virus abundance at 72 hours post-infection (hpi) ${ }^{22}$. To further investigate the mechanisms of dsRNA triggered antiviral defense and the dynamics of virus infection and corresponding immune responses in honey bees, we performed a time series experiment (i.e., 6 , 48, and $72 \mathrm{hpi}$ ) that included transcriptional profiling of individual virus-infected and dsRNA-treated bees. We determined that honey bee gene expression varied with the progression of virus infection and included genes involved in endocytosis, development, transcriptional regulation, RNAi, and the Toll, Imd, JAK-STAT, and JNK pathways. Interestingly, bees that exhibited decreased virus abundance in the context of dsRNA treatment exhibited increased expression of two RNA helicases, one JNK pathway member, and several genes involved in dsRNA transport. Furthermore, we performed in vivo studies that confirmed the importance of the genes dicer and cyclin-dependent serine/threonine kinase (MF116383), which exhibited increased expression in virus-infected bees, in honey bee antiviral defense. Together these results further our understanding of honey bee antiviral defense mechanisms and the effects of dsRNA on honey bee gene expression and may lead to the development of strategies that limit virus infection in honey bees. Development and increased use of siRNAs and dsRNAs to reduce pathogens and pests (i.e., fungi, nematodes, and insects) in crops that are frequently visited by pollinators $^{50,51}$ also underscores the need to further examine the effects of these molecules on bee health.

\section{Results and Discussion}

Honey bees are commonly infected by positive sense single-stranded RNA (+ssRNA) viruses, which replicate via a dsRNA intermediate. RNAi mediated by virus-specific dsRNA is an important antiviral defense mechanism in honey bees ${ }^{9,22,36,38-41}$, but nonspecific dsRNA-triggered antiviral response pathway(s) also play a role in honey bee antiviral defense ${ }^{22}$. To further investigate honey bee antiviral defense mechanisms, including RNAi and nonspecific dsRNA-mediated mechanisms ${ }^{22}$, we infected bees with a model virus in the presence or absence of dsRNA (Fig. 1). There are currently no honey bee virus isolates or infectious clones, so we utilized a recombinant Sindbis virus that expresses GFP (SINV-GFP) as a tractable model of virus infection in honey bees ${ }^{22}$. SINV-GFP inoculations were performed via intrathoracic injection in the absence or presence of multiple species and lengths of dsRNA, including virus-specific dsRNA ( $s p-d s R N A, 928 \mathrm{bp}$ ), nonspecific dsRNA corresponding to Drosophila $\mathrm{C}$ virus sequence (ns-dsRNA, 1,017 bp), and luciferase sequence (dsRNA-short, $355 \mathrm{bp}$ ). In addition, co-administration of polyinosinic-polycytidylic acid (poly(I:C)), a synthetic mimic of dsRNA, or nucleoside triphosphates (NTPs), served as positive and negative controls, respectively; mock-infected controls were also 
A

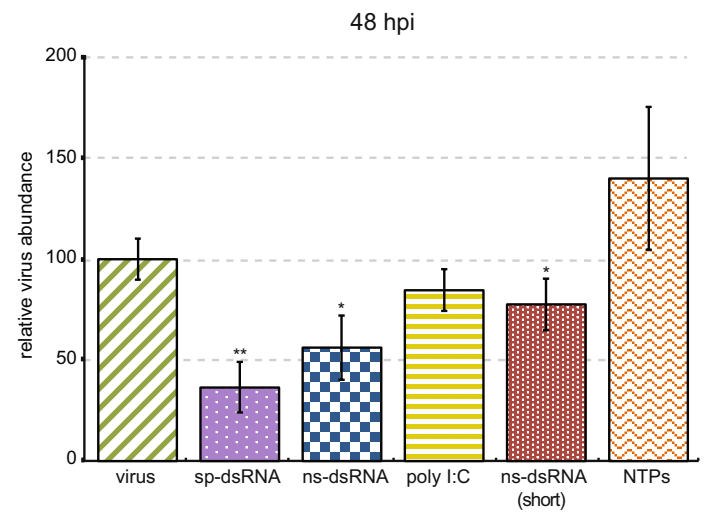

B

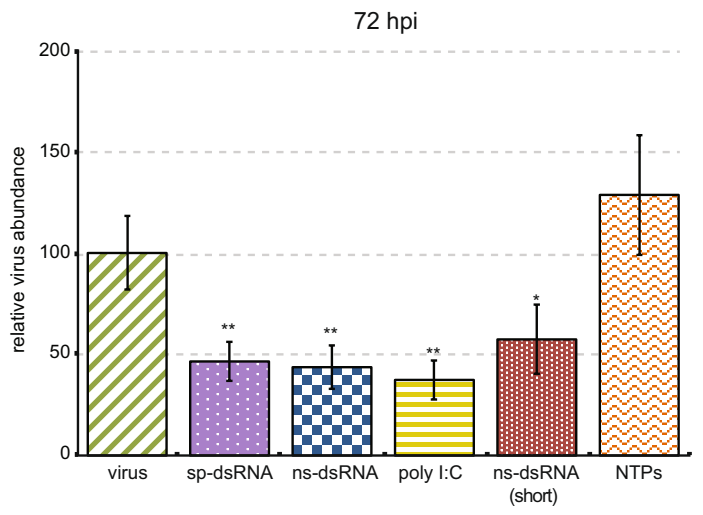

Figure 2. Relative virus RNA abundance was reduced in dsRNA-treated bees as compared to virus-infected bees. Relative abundance of SINV-GFP in individual bees $(n=10)$ was assessed by qPCR. (A) At 48 hours post-infection (hpi), bees treated with dsRNA $(1 \mathrm{~kb})$ had reduced relative virus RNA abundance (includes both virus genomes and transcripts) by $64 \%$ for sp-dsRNA-treated bees (dotted purple, $* *$ p $<0.005$ ) and $44 \%$ for nsdsRNA-treated bees (checkered blue, $* \mathrm{p}<0.05$ ), as compared to bees infected with virus only (green stripes). Likewise, bees treated with short dsRNA $(0.5 \mathrm{~kb})$ had $24 \%$ less virus (dotted red, $* \mathrm{p}<0.05)$ than virus-infected bees. (B) At 72 hpi bees treated with dsRNA had reduced relative virus abundance by $54 \%(* * \mathrm{p}<0.005)$ and $56 \%(* * \mathrm{p}<0.005)$ for sp-dsRNA and ns-dsRNA, respectively. Similarly, bees treated with poly(I:C) (yellow stripes), a structural analog of dsRNA, had $63 \%$ less virus than virus-infected bees $(* * \mathrm{p}<0.005)$. Bees treated with short dsRNA also had $43 \%$ less virus (dotted red, ${ }^{*} \mathrm{p}<0.05$ ) than bees infected with virus alone. The virus abundance in bees treated with NTPs (orange wavy lines) was not significantly different from virusinfected bees at either $48 \mathrm{hpi}$ (A) or $72 \mathrm{hpi} \mathrm{(B).} \mathrm{Percent} \mathrm{relative} \mathrm{virus} \mathrm{abundance} \mathrm{for} \mathrm{each} \mathrm{sample} \mathrm{was} \mathrm{determined}$ via $\Delta \Delta \mathrm{CT}$ analysis (using $A m$ rpl8 as the house keeping gene); statistical differences between treatment and virus-infected bees were performed using one-sided Student's t-tests, ${ }^{*} \mathrm{p} \leq 0.05,{ }^{*} \mathrm{p} \leq 0.005$. The bars represent the standard error of the mean.

performed. Bees from each experimental treatment group were collected at 6,48 , and $72 \mathrm{hpi}$, a time course that allowed for assessment of both early and late antiviral responses. Relative virus abundance was examined via fluorescence microscopy and quantified based on relative protein and RNA abundance using Western blot analyses and quantitative PCR (qPCR), respectively (Figs 1 and 2 and S1 and S2).

Virus abundance reduced in dsRNA-treated honey bees. Honey bees treated with sp-dsRNA or ns-dsRNA had reduced virus abundance. Fluorescent microscopy of bees that were infected with SINV-GFP in the absence or presence of dsRNA provided qualitative evidence that dsRNA treatment reduced SINV-GFP at 72 hpi (Supplementary Fig. S1). To more quantitatively examine the reduction of SINV-GFP, we performed Western blot analyses of individual bee lysates at $72 \mathrm{hpi}$, which determined that dsRNA, regardless of sequence composition, reduced virus abundance at the protein level (Supplementary Fig. S2). Virus abundance was most accurately measured and compared by assessing relative RNA abundance via qPCR (Supplementary Table S1 and Supplementary Fig. S3). At 48 hpi and 72 hpi, bees treated with sp-dsRNA, ns-dsRNA, and poly(I:C) had decreased relative SINV-GFP abundance as compared to bees infected with virus only and bees simultaneously treated with virus and NTPs (Figs 2 and S4). At 48 hpi, the relative virus abundances of sp-dsRNA and ns-dsRNA treated bees were reduced by $64 \%(\mathrm{p}<0.005)$ and $44 \%(\mathrm{p}<0.05)$, respectively, as compared to bees infected with virus only (Fig. 2A). At $72 \mathrm{hpi}$, the relative virus abundances of sp-dsRNA- and ns-dsRNA-treated bees were reduced by $54 \%$ $(\mathrm{p}<0.005)$ and $56 \%(\mathrm{p}<0.005)$ as compared to bees infected with virus only (Fig. $2 \mathrm{~B})$. Bees treated with poly(I:C) at $72 \mathrm{hpi}$ had reduced virus abundance by $63 \%(\mathrm{p}<0.005)$. Reduced relative virus abundance in dsRNA-treated bees was also observed in additional biological replicates, which included virus-infected bees at 48 and $72 \mathrm{hpi}$ from two additional honey bee colonies for a total of $n=30$ per treatment (Supplementary Fig. S4).

Transcriptional level evaluation of virus and dsRNA induced immune responses in honey bees. The transcriptional profiles of virus-infected honey bees are indicative of the cellular pathways and mechanisms that are regulated in response to virus infection. Likewise, we hypothesized that a subset of the differentially expressed genes would also be regulated in response to dsRNA, a VAMP. To further elucidate the honey bee transcriptional response to virus infection and the mechanisms of dsRNA triggered antiviral defense, we performed transcriptome profiling (RNASeq) of individual virus-infected bees, bees infected with virus in the presence of sp-dsRNA or ns-dsRNA, dsRNA-treated bees in the absence of virus, and mock-infected bees at 6, 48, and 72 hpi (Fig. 1). Forty-seven individual bee RNASeq libraries were prepared using the Illumina TruSeq Stranded RNA Sample Prep kit and paired-end sequenced $(2 \times 100 \mathrm{nt})$ on an Illumina HiSeq. 2500, resulting in an average of 12 million reads per individual bee sample (Supplementary Table S2). On average, 77\% of reads mapped to the $A$. mellifera genome assembly 4.5 from $\mathrm{NCBI}^{52}$. Prior to sequencing, bees were screened for confounding 
pre-existing infections via pathogen-specific PCR and qPCR in order to identify individuals with little to no preexisting infections (Supplementary Tables S1 and S3).

Genes differentially expressed in virus-infected bees and dsRNA-treated bees. Transcriptome analysis of virus-infected bees over the course of infection (i.e., 6, 48, and $72 \mathrm{hpi}$ ) determined that virus-infection altered the expression of hundreds of genes as compared to mock-infected bees (Figs 3A and S5). The majority of differentially expressed genes (DEGs) in virus-infected and dsRNA-treated bees are not well characterized or do not have known roles in antiviral defense (Supplementary Table S5). Genes that exhibited increased expression at $6 \mathrm{hpi}$ were functionally enriched for the biological processes phosphorylation and transcriptional regulation (Supplementary Fig. S6). The genes with increased expression at $48 \mathrm{hpi}$ were enriched in transcriptional regulation, cell adhesion, immune responses, and cellular migration (Supplementary Fig. S6 and Supplementary Table S5). Similarly, virus-infected bees $72 \mathrm{hpi}$ also exhibited increased expression of genes enriched for transcriptional regulation and gene silencing (Supplementary Fig. S6). Genes involved in morphogenesis were differentially expressed throughout all time points (Supplementary Fig. S6) ${ }^{53}$.

Venn diagram analysis demonstrated that the honey bee transcriptional response to virus infection varies with time post-infection (Fig. 3A). As time post-infection increased, so did the number of DEGs, from 236 DEGs to 773 DEGs (Fig. 3A). Twenty-three genes were commonly differentially expressed throughout the course of the infection (Fig. 3A), though only eight of these genes were differentially expressed in a uniform direction (Fig. 3A and Supplementary Tables S4, S5 and S7). Six of these genes exhibited increased expression including an uncharacterized transcript encoding a probable cyclin-dependent serine/threonine kinase (MF116383), apid1, DNA n6-methyl adenine demethylase (loc412878), orb2-like, solute carrier organic anion transporter family member 3a1-like (sloc3a1), and titin-like (Fig. 3A and Supplementary Tables S4, S5 and S7). Two genes had lower expression in all virus-infected bees: obp16 and zinc finger protein 431-like.

Many viruses generate long dsRNA molecules during their replication cycle. Long dsRNA molecules are not a typical product of eukaryotic gene expression, so they serve as triggers of eukaryotic antiviral immune responses (e.g., RNAi and interferon responses) ${ }^{46,54}$. To further investigate the role of dsRNA stimulation in honey bee antiviral defense, we examined changes in gene expression over time. The genes that exhibited increased expression in dsRNA treated bees 48 hours after treatment were enriched for functions including oxidation-reduction, cellular morphogenesis, and immune response (Supplementary Table S5). Bees 72 hours post-treatment exhibited increased expression of genes enriched for cellular morphogenesis, transcriptional regulation, vesicle-mediated transport, and RNA interference (Supplementary Table S5), paralleling the results of a previous study that examined the effects of nonspecific dsRNA (GFP-dsRNA) on honey bee gene expression ${ }^{43}$.

Venn diagram analysis of dsRNA-treated bees 6,48 , and 72 hours post-treatment identified 14 shared DEGs, three of which exhibited decreased expression: carbonic anhydrase 1, venom acid phosphatase acph-1-like, and odorant binding protein 16, which also exhibited decreased expression in virus-infected bees (Fig. 3B, Supplementary Tables S5 and S8). Similar to virus-infected bees, heat shock protein 90 ( $h s p 90$ ) was also differentially expressed throughout all dsRNA-treated bees. In dsRNA-treated bees 6 and 48 hpi, several genes encoding antimicrobial peptides (i.e., apidaecin, apidaecins type 73-like, abaecin and hymenoptaecin) exhibited increased expression (Fig. 3B), most of which also exhibited increased expression in virus-infected bees (Supplementary Table S6). In addition, both virus-infected and dsRNA-treated bees 48 and 72 hpi exhibited increased expression of scavenger receptor class $c(s c r-c)$, which plays a role in dsRNA uptake in D. melanogaster ${ }^{55}$ and may play an analogous role in honey bees. Together, our analyses indicate that dsRNA-treatment alters gene expression in honey bees, and that there are common and unique aspects between differential gene expression in virus-infected bees and virus-infection in the context of dsRNA.

qPCR validation of RNASeq results. In order to validate RNAseq results, we examined the relative expression of fourteen genes (i.e., cyclin-dependent kinase, orb2-like, titin-like, DNA n6-methyl adenine demethylase, slco31-like, hsp90, abaecin, ago2, dicer, igfn3-10, mfs-transporter, jra, fam 102b) that exhibited increased expression in virus and/or dsRNA treated bees at 48 and/or 72 hpi via qPCR of sequenced bees 72 hpi (Supplementary Fig. S7). The expression of ten of those genes was also examined by qPCR in sequenced bees at 48 hpi (Supplementary Fig. S7). All but two of the fourteen genes assayed (igfn3-10 and titin-like) were confirmed to have increased expression in virus-infected and/or dsRNA-treated bees via qPCR (Supplementary Fig. S7). Several genes (e.g., hsp90, cyclin-dependent kinase, ago2, dicer, $m f$ s-transporter, formin-j) were also confirmed to have increased expression in biological replicate experiments that utilized pooled virus-infected honey bee samples ( $72 \mathrm{hpi})$ from two different colonies, likely with different genetic backgrounds (Supplementary Fig. S8). Together these results confirm the RNASeq results and provid further evidence to their importance in honey bee antiviral defense.

Differentially expressed genes in a cellular context. To compare our results with what is currently known about insect immunity, we surveyed the DEGs of virus-infected and dsRNA-treated bees for genes involved in previously characterized insect immune pathways. This analysis determined that many genes encoding extracellular receptors and proteins involved in endocytosis, signal transduction, as well as immune effector proteins (e.g., antimicrobial peptides) exhibited increased expression (Fig. 4 and Supplementary Table S6). Some of the genes identified herein are illustrated in a cellular context in order to illustrate their potential functions in antiviral defense (Fig. 4).

Extracellular receptors and transporters. In the context of virus infection, extracellular receptors can serve in pathogen recognition and host defense or be co-opted by the virus to facilitate entry. The membrane localized solute carrier organic anion transporter family member 3a1-like (sloc3a1) consistently exhibited increased expression in virus-infected bees (i.e., 1.6-2.3 fold increase) (Figs 3A and 4 and Supplementary Tables S4, S5 and S7). 

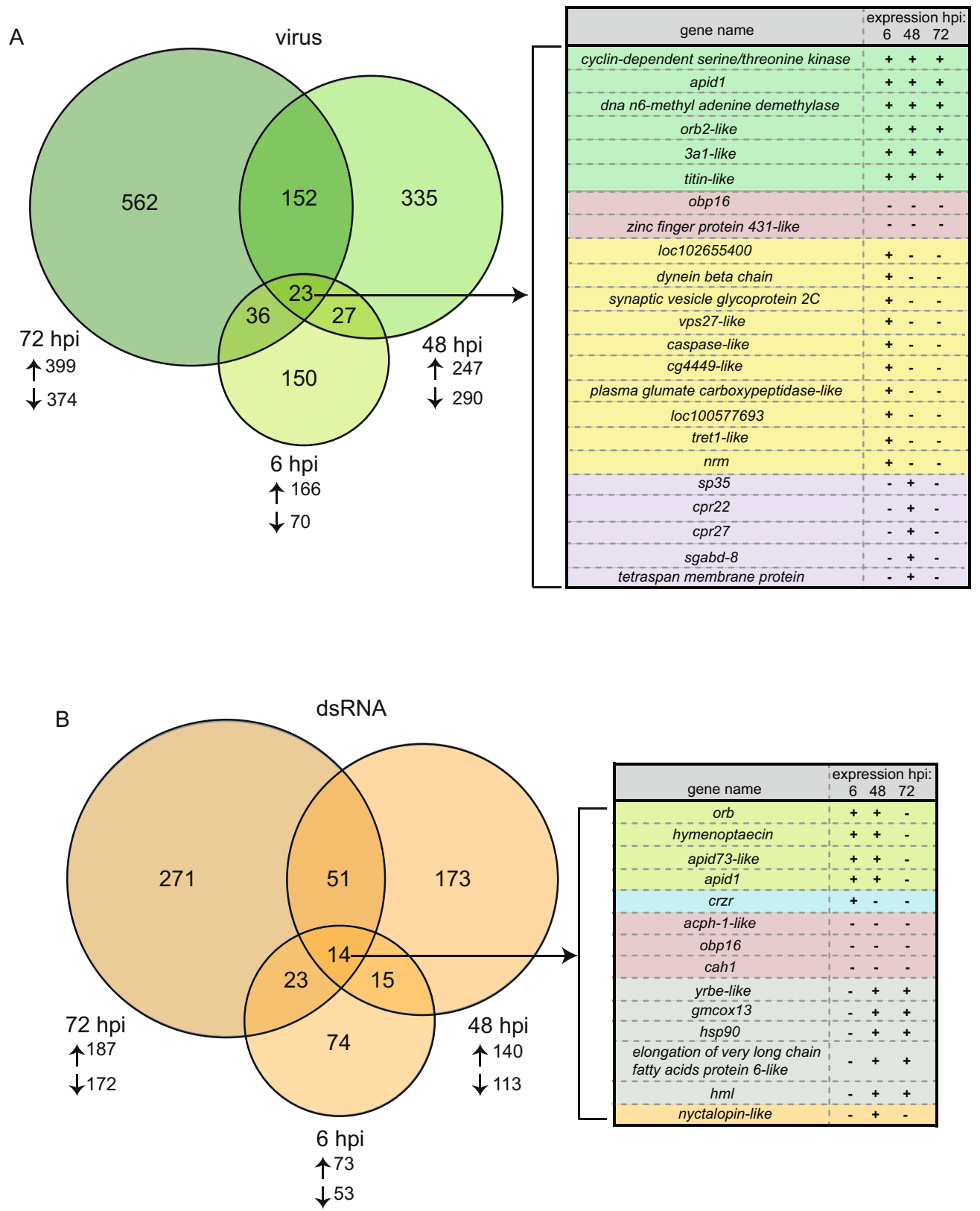

Figure 3. Honey bee transcriptional response to virus-infection and dsRNA-treatment is time-dependent. (A) There were hundreds of differentially expressed genes (DEGs) in virus-infected bees, as compared to mock-infected bees. Venn diagram analysis identified shared and unique DEGs of virus-infected bees 6, 48, and 72 hours post-infection (hpi). Twenty-three genes were differentially expressed at all three time points post-infection. Six of these genes consistently exhibited increased expression (highlighted in green and listed from highest average $\log _{2}$ fold change to lowest) and two genes consistently exhibited decreased expression (red). Ten genes exhibited increased expression at $6 \mathrm{hpi}$ and decreased expression at 48 and $72 \mathrm{hpi}$ (yellow). Five genes (purple) displayed increased expression at $48 \mathrm{hpi}$, but decreased expression at 6 and $72 \mathrm{hpi}$. Arrows pointing up denote number of genes that exhibited increased expression for each time point and arrows pointing down denote number of genes that exhibited decreased expression. (B) There were hundreds of differentially expressed genes (DEGs) in dsRNA-treated bees compared to mock-infected bees. Venn diagram analysis identified shared and unique DEGs of dsRNA-treated bees at 6, 48, and $72 \mathrm{hpi}$, including 14 genes that were differentially expressed at all time points. There were four genes (green) that exhibited increased expression at 6 and $48 \mathrm{hpi}$ and decreased expression at $72 \mathrm{hpi}$, three of which are antimicrobial peptides (AMPs). One gene, $c r z r$, exhibited increased expression at $6 \mathrm{hpi}$, but decreased expression in bees 48 and $72 \mathrm{hpi}$ (blue). Three genes displayed consistently decreased expression (red). Five genes had decreased expression at $6 \mathrm{hpi}$ and increased expression at 48 and $72 \mathrm{hpi}$ (gray), including heat shock protein 90. One gene, nyctalopin-like, exhibited decreased expression in bees 6 and $72 \mathrm{hpi}$, but increased expression in bees $48 \mathrm{hpi}$ (orange). DEGs with Benjamini-Hochberg corrected q-values $\leq 0.05$ were included in Venn diagram analyses. Full lists of DEGs and their fold changes from all contrasts in each Venn diagram are provided in Supplementary Tables S7 and S8. 


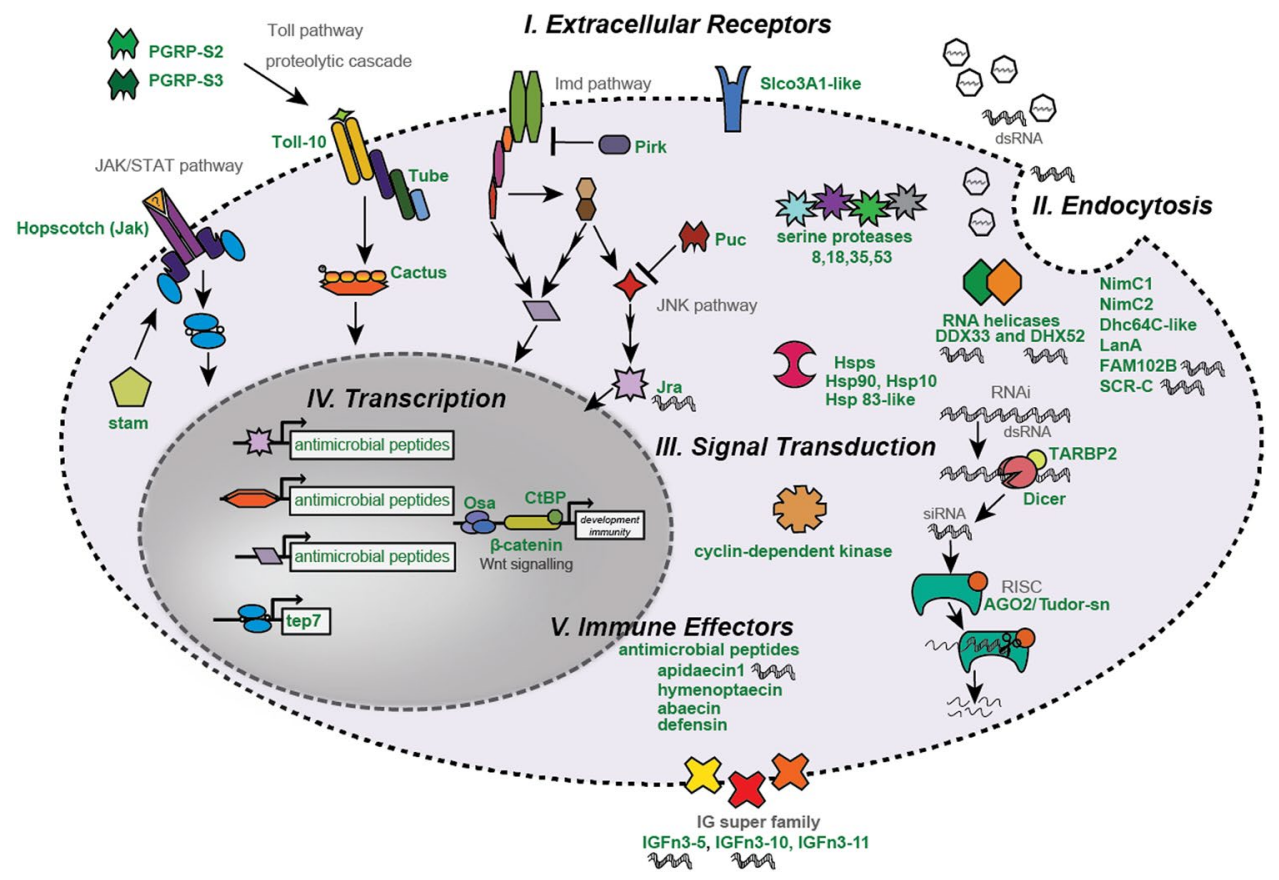

Figure 4. Differentially expressed genes in response to virus and/or dsRNA treatment in a cellular context. The DEGs of virus-infected and dsRNA-treated honey bees were surveyed for differential expression of genes involved in previously characterized insect immune pathways: RNAi, Toll, JAK/STAT (Janus Kinase and Signal Transducer and Activator of Transcription), Imd (Immune Deficiency), and JNK (c-Jun N-terminal kinases) ${ }^{29,56}$. This analysis determined that many genes encoding extracellular receptors, proteins involved in endocytosis, signal transduction, and immune responses exhibited increased expression; these DEGs (denoted by bold and green font) are depicted in a cellular context. Many genes exhibited higher fold change in bees treated with both virus and dsRNA as compared to bees infected with virus only (denoted with dsRNA), suggesting their involvement in dsRNA-triggered immune responses. Complete DEG lists are provided in Supplementary Table S6.

Interestingly, slco3a1 also exhibited increased expression in SBV and DWV infected bees ${ }^{25}$. The JAK/STAT receptor, hopscotch, exhibited increased expression in virus-infected bees (Fig. 4 and Supplementary Table S6). The JAK/STAT pathway is involved in both insect development and antiviral defense ${ }^{29,56}$. Additionally, two pathogen recognition receptors of the Toll pathway, peptidoglycan receptor s2 (pgrp-s2) and peptidoglycan receptor $s 3$ (pgrp-s3), and the toll-like receptor toll-10 exhibited increased expression in virus-infected bees (Fig. 4 and Supplementary Table S6). Similar to virus-infected bees, bees treated with only dsRNA also exhibited greater expression of toll-10 and pgrp-s3. The Toll pathway is primarily involved in defense against Gram-positive bacteria and fungi, but has also been implicated in antiviral defense in D. melanogaster and Aedes aegypti $i^{56}$. Similar to the Toll pathway, activation of the Imd pathway results in antimicrobial peptide production, but it is typically activated by Peptidoglycan recognition protein LC (PGRP-LC) binding to the diaminopimelic-containing peptidoglycan of Gram-negative bacteria ${ }^{29}$. The Imd pathway is also important for fruit fly antiviral defense against some viruses, including Sindbis virus ${ }^{56-58}$.

Lastly, three genes encoding immunoglobulin domain containing proteins (i.e., igfn3-5, igfn3-10, and igfn3-11) exhibited increased expression in virus-infected bees (Fig. 4 and Supplementary Table S6). In insects, immunoglobulin domain containing proteins serve in a variety of functions, including cell to cell adhesion, pathogen recognition, and promotion of phagocytosis ${ }^{59}$. Similarly, hemolin, an immunoglobulin-domain containing protein exclusively expressed in lepidopterans, exhibits increased expression in Chinese Oak Silk moths treated with dsRNA or infected by Baculovirus ${ }^{60}$.

Endocytosis. Endocytosis, including phagocytosis, is an immune effector function carried out by hemocytes ${ }^{61}$ and may also be important for systemic RNAi ${ }^{55,62}$. In addition, many viruses exploit endocytic pathways for entry $^{63}$. SINV and other alphaviruses typically enter cells via receptor binding followed by clathrin-mediated endocytosis ${ }^{64}$. Several genes involved in phagocytosis, including nimc1, nimc2, dhc64c-like, and laminin a, exhibited increased expression in virus-infected bees (Fig. 4 and Supplementary Table S6). Laminins aid in cellular adhesion, migration, differentiation, and morphology ${ }^{65}$. In mammalian cells, SINV utilizes a laminin receptor for viral entry ${ }^{65}$. Likewise, virus-infected honey bees had greater expression of a JAK/STAT effector molecule thioester protein 7 (tep7). In mosquitoes, thioester proteins bind to invading bacteria which promotes phagocytosis of these pathogens ${ }^{66}$, but thioesters are also associated with improved defense against Dengue and West Nile viruses via unknown mechanisms ${ }^{67}$.

In D. melanogaster $\mathrm{S} 2$ cells, genes involved in receptor-mediated endocytosis are important for dsRNA uptake, including the genes scavenger receptor $c(s c r-c)$, fam 102b, and $s a p-r^{55}$. Bees treated with virus, dsRNA, or both 
exhibited increased expression of $s c r-c$, but bees treated with both virus and dsRNA exhibited the greatest increase (Fig. 4 and Supplementary Table S5). Additionally, the expression of fam $102 b$ was significantly increased in bees that were treated with both virus and dsRNA. In contrast, sap- $r$, which is a protease associated with late stage endosomes, exhibited decreased expression in bees treated with both virus and dsRNA and may be indicative of virus-specific dsRNA triggered modification of endosomal development ${ }^{68}$, although future investigation is required.

Signal transduction cascades. Signal transduction cascades are the means by which a chemical or physical signal is transmitted through a cell resulting in a response. For example, detection of pathogen associated molecular patterns (PAMPs), including dsRNA, results in activation of cellular transduction cascades that activate particular immune responses ${ }^{69}$. In our data set, reads aligning to Apis mellifera LOC25387, which encodes a previously uncharacterized transcript (MF116383) that has high sequence homology to an Eastern honey bee (Apis cerana) probable cyclin-dependent serine/threonine kinase (XM_017051141.1), exhibited the greatest increase in expression in virus-infected bees as compared to mock-infected controls (i.e., 5.7-13 fold increase) (Figs. 3A and 4, Supplementary Fig. S9, and Supplementary Tables S4, S5, and S7) ${ }^{9}$. In general, cyclin-dependent serine/threonine kinases are activated by cyclins and phosphorylate serine and threonine residues of substrate proteins, resulting in regulation of cell cycle progression and transcription. Though, the specific proteins that interact with this cyclin-dependent serine/threonine kinase are unknown. Likewise the expression of Am LOC25387 transcripts were increased in DWV and SBV co-infected bees and IAPV-infected bees ${ }^{9,25}$.

Genes involved in Toll pathway signal transduction also exhibited increased expression in virus-infected bees including cactus 1 and cactus 2, which suppress NF- $\kappa B$ signaling, and tube, an adaptor protein that promotes NF- $\kappa$ B signaling (Fig. 4 and Supplementary Table S5). In Drosophila, immune pathways are tightly regulated in order to balance immune responses, thus increased expression of pathway inhibitors (e.g., cactus 2) does not necessarily indicate complete or continuous repression of the pathway (e.g., Toll $)^{70}$. Likewise, we determined that pirk, which represses Imd pathway signaling ${ }^{71,72}$, exhibited increased expression in virus-infected bees (Fig. 4 and Supplementary Table S5). Intriguingly, JNK pathway activation is often linked with Imd pathway activation ${ }^{56}$. The transcriptional effector of the JNK pathway, jun-related antigen ( $\mathrm{ra}$ ), had greater expression in bees that were both virus-infected and treated with dsRNA at 6 and $48 \mathrm{hpi}$, and increased expression in all virus-infected groups at 72 hpi (Supplementary Fig. S7 and Supplementary Table S5). Bees treated with only dsRNA followed similar, but lower, expression patterns as compared to mock-infected bees, suggesting that the JNK signaling may be involved in dsRNA-triggered responses.

The Wnt/beta-catenin signaling pathway, which is involved in cellular proliferation and differentiation, has also been implicated in insect host-virus interactions, though its role in immune function is less well characterized $^{73,74}$. Several genes involved in Wnt signaling (e.g., osa) exhibited increased expression in virus-infected bees (Fig. 4 and Supplementary Table S5). The involvement of the Wnt signaling pathway in honey bee antiviral defense has also been implicated in the context of IAPV infection ${ }^{9}$, thus it is likely that Wnt signaling is important to honey bee antiviral defense.

Immune effector proteins. Several antimicrobial peptides, which are effector molecules of Toll, Imd, and JNK pathways, exhibited increased expression in virus-infected and dsRNA-treated bees. Importantly, apidaecin 1 exhibited increased expression in all virus-infected bees and bees treated with dsRNA alone (Figs 3 and 4 and Supplementary Tables S4-S8) ${ }^{56,75}$. Apidaecins are proline-rich antimicrobial peptides (AMPs) that have bactericidal activity against Gram-negative bacteria ${ }^{75,76}$. Virus-infected and/or dsRNA-treated bees also exhibited increased expression of abaecin and hymentoptaecin (Supplementary Table S5), indicating activation the Imd and/ or JNK pathways ${ }^{56,75,77}$. Increased AMP expression in virus-infected honey bees and other insects has previously been reported, though their role in antiviral defense is not yet understood ${ }^{24-26,78}$. It may be that AMPs do not have a direct role in antiviral defense and that increased transcript levels of AMPs and genes involved in pathogen recognition and signal transduction (e.g., pgrp-s3) simply indicate activation of these pathways ${ }^{56,75,79}$. The activation of Toll, Imd, and JNK signal transduction cascades likely stimulate transcription of hundreds of genes, including antiviral effectors that await further characterization.

Heat shock proteins (Hsps) are involved in general stress responses and protein degradation and stabilization. In fruit flies, these ubiquitously expressed proteins are important for defense against some viruses ${ }^{80,81}$. Our transcriptional level analysis identified several genes encoding heat shock and accessory proteins that exhibited increased expression in virus-infected bees including hsp90, activator of hsp90, $60 \mathrm{kda} h s p, 10 \mathrm{kda}$ hsp, hsp83-like, and $h s f 5$ (Supplementary Table S5). Hsp90 expression was also increased in dsRNA-treated bees. In Drosophila, Hsp90 binds to and stabilizes the RNA-induced silencing complex (RISC) as part of the RNAi response ${ }^{82,83}$, but Hsp90 can also be exploited by both insect and human viruses (e.g., Flock House virus and Polio virus) in order to stabilize RNA replication ${ }^{84,85}$. Future studies aimed at better understanding the functions of heat shock proteins, particularly Hsp90, in virus-infected honey bees will be exciting since these proteins may either be antagonistic or beneficial to specific viruses.

RNA interference is an important antiviral and post-transcriptional gene regulatory mechanism in honey bees that is initiated by Dicer recognition of dsRNA $22,36,37,39$. Notably, there was greater expression of genes involved in RNAi (i.e., argonaute-2 (ago2), dicer, tudor-sn, hsc70-4, and tarbp2) in virus-infected bees (Fig. 4 and Supplementary Table S6). Interestingly, enhanced expression of dicer and ago-2 in virus-infected honey bees was observed in another study ${ }^{24}$, whereas increased expression of genes involved in RNAi has not been observed in virus-infected fruit flies ${ }^{49}$. In our studies, administration of dsRNA, in the absence of virus infection, did not induce Apis mellifera dicer or ago2 expression, indicating that VAMP immune triggering does not completely recapitulate the immune response to virus infection. Additional studies are required to better understand the mechanisms of transcriptional activation of genes involved in honey bee $\mathrm{RNAi}^{86}$. 


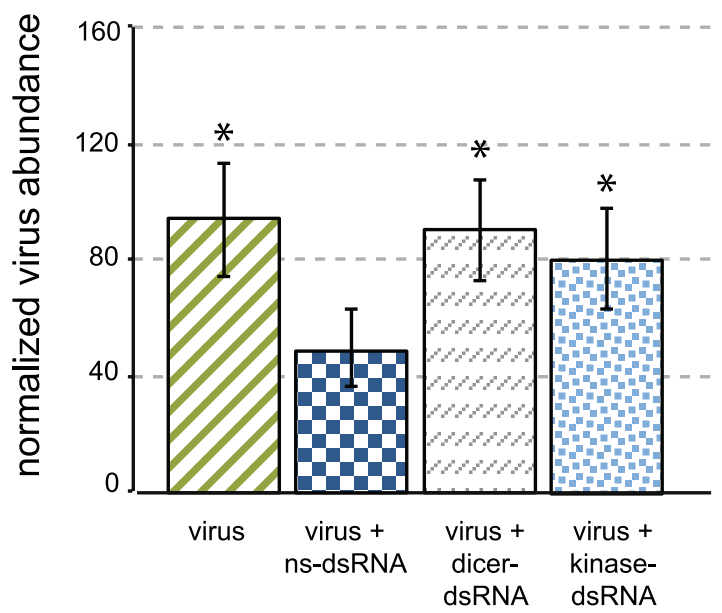

Figure 5. Reduced expression of two honey bee genes resulted in increased virus abundance. To further investigate the role of dicer and cyclin-dependent kinase in honey bee antiviral defense, we utilized RNAimediated gene knock down to reduce their expression (Supplementary Fig. S10) and qPCR to determine the impact on virus abundance. SINV-GFP abundance in kinase and dicer specific dsRNA-treated bees at $72 \mathrm{hpi}$ was increased by $48 \%(* \mathrm{p}<0.05)$ and $44 \%(* \mathrm{p}<0.05)$, respectively, compared to the ns-dsRNA control, which is the most relevant comparison given that administration of dsRNA, including the dsRNAs used to reduce the expression of dicer and cyclin-dependent kinase reduces virus abundance. Percent relative virus abundance for each sample was determined via qPCR and $\Delta \Delta \mathrm{CT}$ analysis using Am rpls as the house keeping gene. Statistical differences between the ns-dsRNA control group and the other treatments were determined using one-sided Student's t-tests, $* \mathrm{p} \leq 0.05, * * \mathrm{p} \leq 0.005$. The bars are standard error of the mean.

The role of DExD box RNA helicases in honey bee antiviral defense is particularly interesting because in mammals many of these proteins function as nonspecific cytosolic sensors of dsRNA (e.g., MDA-5 and RIG-I), which activate the antiviral interferon response ${ }^{46,49}$. In Culex pipiens $f$. molestus mosquitoes, D. melanogaster, and Bombus terrestris, Dicer-2 serves as a dsRNA pathogen recognition receptor (PRR), that after binding dsRNA, results in the increased activation of antiviral immune effectors (e.g., vago) ${ }^{29,42,44,47,49}$. DWV-infected honey bees exhibited increased expression of Apis mellifera vago (loc503505), but differential vago expression was not observed in our data set; many host factors (e.g., age/life stage) may be involved, but perhaps vago expression is only increased in response to specific honey bee infecting viruses (Supplementary Table S5). The expression of two RNA helicases (i.e., rna helicase $d d x 33$ and rna helicase $d h x 52$ ) was increased in virus-infected and dsRNA-treated honey bees (Fig. 4 and Supplementary Table S5). RNA helicase DHX33 has been identified as a dsRNA receptor in mammals that when bound to dsRNA or bacterial RNA, activates NLRP3 inflammasome-mediated interferon stimulation ${ }^{87}$, but RNA Helicase DDX33 has not been implicated in dsRNA-immunostimulation in insects. Future exploration of the role of these important dsRNA sensors in activating antiviral response in honey bees will likely lead to the discovery of analogous pathways in other organisms.

Reduced expression of dicer and cyclin-dependent kinase enhanced virus abundance in vivo and confirmed their role in limiting virus infection in honey bees. In order to further investigate the biological importance of two putative antiviral genes, dicer and a probable cyclin-dependent serine/threonine kinase (MF116383), we utilized RNAi-mediated gene knock down to reduce their expression and investigate the impact on virus abundance. We expected that reduced expression of these antiviral genes would result in increased virus abundance, as compared to the virus abundance in bees treated with ns-dsRNA. Virus-infected bees treated with cyclin-dependent kinase-specific dsRNA exhibited decreased expression by 48 hpi (40\%) and $72 \mathrm{hpi} \mathrm{(30 \% )} \mathrm{com-}$ pared to respective controls (i.e., virus-infected and ns-dsRNA treated bees) (Supplementary Fig. 10). At $72 \mathrm{hpi}$, virus abundance in bees with reduced cyclin-dependent kinase levels was higher compared to the virus abundance in bees treated with ns-dsRNA, $77 \%$ versus $43 \%$ relative virus abundance $(\mathrm{p}<0.05)$ (Fig. 5). Bees 48 hpi followed similar trends (Supplementary Fig. 10). Bees treated with dicer-specific dsRNA in the context of virus infection exhibited reduced expression of dicer at $48 \mathrm{hpi}$, but not $72 \mathrm{hpi}$ (Supplementary Fig. 10). Though the kinetics of dicer knock-down differed from the probable cyclin-dependent kinase, bees treated with dicer specfic dsRNA had a greater abundance of virus compared to bees treated with ns-dsRNA, $90 \%$ versus $43 \%$ (Fig. 5). These results confirm the role of dicer in limiting virus infections in honey bees and highlight the importance of a previously uncharacterized transcript encoding a probable kinase, cyclin-dependent kinase (MF116383), in limiting virus infection. Further investigation of this probable cyclin-dependent kinase and the proteins with which it interacts may lead to the discovery of novel honey bee antiviral pathways or aid in further characterization of known immune pathways.

Synthesis of honey bee transcriptional response to virus infection. This is the first study to examine individual honey bee antiviral responses to infection with controlled inoculum of a model virus at multiple time points. Other studies have examined honey bee responses to virus infection at the transcriptional level, but 


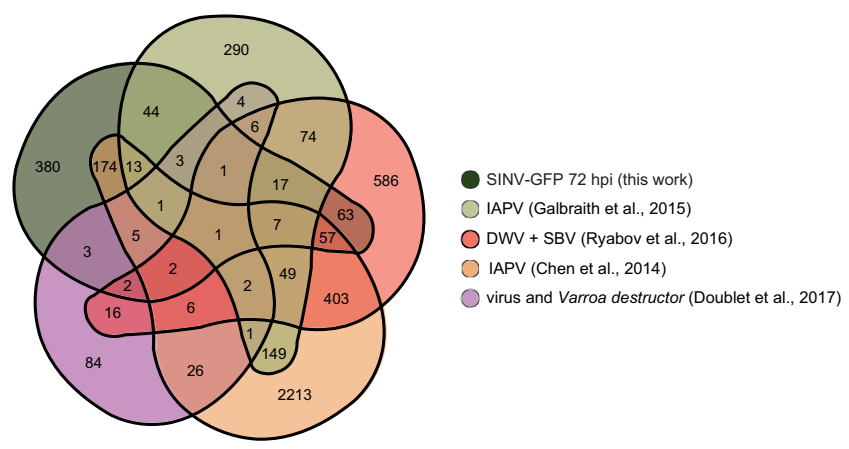

Figure 6. Venn diagram of shared and unique DEGs in bees infected with viruses from this and other studies. The DEGs identified in SINV-GFP-infected bees $72 \mathrm{hpi}$ were compared to those identified in naturally IAPVinfected bees (Chen et al. 2014, orange) ${ }^{9}$, bees infected with IAPV via oral inoculation (Galbraith et al., 2015, green $)^{24}$, SBV and DWV-infected bees (Ryabov et al., 2016, pink) ${ }^{25}$, and a common DEG list that was compiled from 19 gene expression data sets including Varroa destructor-parasitized and virus-infected bees (Doublet et al., 2017) $)^{92}$ (purple). Lists of DEGs from all studies that were used to generate the Venn diagram ${ }^{131}$ are provided in Supplementary Tables S14 and Venn diagram results are listed in S15.

they vary by virus-challenge methodologies (e.g., mite vectored, infection via injection, and oral infection), purity and strain of virus inoculum, tissues examined, post-infection assay time, and bee developmental stage, which reduce commonalities in transcriptional results between studies ${ }^{9,15,22,24-26,88-91}$.

In spite of the methodological differences between this and other honey bee transcriptional level analyses, we identified common DEGs associated with virus infected honey bees. Using Venn diagram analysis, we compared our DEG lists to DEGs of symptomatic IAPV-fed bees ${ }^{24}$, SBV and DWV-infected bees ${ }^{25}$, adult honey bees naturally infected with IAPV 9 , and a synthesis of common DEGs that was recently generated from 19 gene expression data sets from Varroa destructor-parasitized and virus-infected bees ${ }^{92}$ (Fig. 6, Supplementary Tables S14 and $\mathrm{S} 15)$. There was one DEG that was shared in all five DEG lists: protein lethal(2)essential for life-like, which encodes a protein in the small heat shock protein (Hsp 20) family, further supporting the role of heat shock proteins in honey bee antiviral defense (Supplementary Table S15) ${ }^{93}$. Additional comparisons, between this study and other transcriptome data, indicated that there were many shared DEGs involved in the Toll, Imd, JAK/STAT, JNK, and RNAi pathways, as well numerous uncharacterized pathways (Supplementary Tables S14 and S15) ${ }^{9}, 22,24,26,94$. There were 87 shared DEGs between virus-infected bees at $72 \mathrm{hpi} \mathrm{(this} \mathrm{work)} \mathrm{and} \mathrm{symptomatic} \mathrm{IAPV-fed} \mathrm{bees}$ (Fig. 6 and Supplementary Table S15), including increased expression of three genes involved in RNAi (i.e., ago2, dicer, and tar rna-binding protein 2) ${ }^{24}$. A few AMPs also exhibited differential expression in many of the DEG lists. For example, hymenoptaecin exhibited differential expression in SINV-GFP-infected bees, DWV and SBV co-infected bees, and in the virus and Varroa destructor DEG synthesis ${ }^{25,92}$ (Fig. 6 and Supplementary Table S15). The gene encoding for Apidaecin exhibited differential expression in SINV-GFP-infected bees, symptomatic IAPV-fed bees, SBV and DWV-infected bees, and adult honey bees naturally infected with IAPV ${ }^{94,25}$. Notably, one of the top ranked genes with decreased expression in the transcriptome synthesis was zinc finger protein 431-like $e^{92}$ (Fig. 6). Our study also determined that this gene had decreased expression in most virus-infected and dsRNA co-treated bees at all time points (Fig. 3). The members of the Pit-Oct-Unc (POU) family have a wide variety of functions primarily involved in the neuroendocrine system ${ }^{95}$. This may correspond with work showing that pathogen infections induce neuronal and behavioral changes (e.g., premature foraging behavior) in honey bees, which may function as a form of social immunity in insect societies ${ }^{96}$.

\section{Summary}

Managed honey bee colonies in the US and parts of Europe have experienced high annual mortality levels (i.e, 33\% average in US since 2006) ${ }^{4,5,11}$. In addition to other factors, several studies indicate that colony losses correlate with high pathogen incidence and abundance ${ }^{7,9-17}$, including infection by (+)ssRNA viruses ${ }^{20,56}$. The outcome of virus infection is influenced by many factors ${ }^{97}$, including virus strain ${ }^{98}$, agrochemical exposure $^{97}$, nutritional status ${ }^{99}$, genetic diversity of the colony ${ }^{88,100-104}$, colony management, mite parasitism ${ }^{15,89}$, 105, co-infections ${ }^{106,107}$, and immune responses at both the colony and individual levels. Honey bee antiviral responses include canonical immune pathways (e.g., Toll, JAK/STAT, Imd, JNK), RNAi, and nonspecific dsRNA-mediated immune pathways, though the relative roles of these pathways and the mechanistic details of honey bee antiviral immune responses are not thoroughly understood.

This is the first study to examine both individual and temporal honey bee transcriptional response to virus infection. Our results further indicate that honey bee antiviral defense includes canonical insect immune pathways, RNAi, and a nonspecific dsRNA-mediated antiviral defense mechanism. Transcriptional analysis of dsRNA-treated bees showed that dsRNA results in increased expression of genes involved in the JNK pathway, RNA helicases, and dsRNA transport, which parallels dsRNA transport and response mechanisms in fruit flies and mammals. While the results described herein implicate the role of numerous genes, several biological processes, and the involvement of Dicer and a probable cyclin-dependent kinase (MF116383), which had greatest sequence similarity with an Apis cerana gene (XM_017051141.1), in honey bee antiviral defense, future studies are required to further elucidate the honey bee antiviral defense network. Better understanding of honey bee 
antiviral defense mechanisms may aid in the development of strategies that reduce honey bee colony losses and furthers our knowledge of antiviral immune responses in insects, which may ultimately reveal evolutionary conserved pathways in other organisms.

\section{Methods \\ Honey bees. Frames of newly emerging bees were obtained from honey bee colonies maintained at Montana State University in Bozeman, MT, USA. Young ( 24hours post-emergence) female worker bees were utilized for experiments. The bees were housed in modified deli-containers at $32^{\circ} \mathrm{C}$ and were provided water and bee candy ${ }^{22,108}$.}

Sindbis virus (SINV-GFP) infection trials. There are currently no infectious honey bee virus clones, but studies with semi-purified honey bee virus preparations have provided valuable information ${ }^{24,25,56}$. We utilized a recombinant model virus, Sindbis virus expressing green fluorescent protein (SINV-GFP) ${ }^{22,109}$. There are several advantages to utilizing this virus including the ability to control the dose of virus inoculum, monitor the progression of virus infection using GFP, and the assurance that the honey bees were not previously infected with, nor exposed to, SINV-GFP. In addition, Sindbis virus does not encode a suppressor of RNAi (VSR) ${ }^{110}$. We and others have used SINV-GFP to investigate honey bee ${ }^{22}$, fruit $\mathrm{fly}^{109}$, and mosquito ${ }^{111}$ antiviral defense mechanisms, thus facilitating comparison of immune responses in both natural mosquito hosts and non-native hosts (i.e., honey bee and fruit fly) that have not co-evolved with this virus. Honey bees were immobilized via incubation at $4^{\circ} \mathrm{C}$ for 20 minutes and injected in the thorax with 3,750 plaque forming units (PFUs) of SINV-GFP 22 diluted in $2 \mu \mathrm{l}$ of $10 \mathrm{mM}$ Tris buffer ( $\mathrm{pH} 7.5)$ using a Harbo large capacity syringe equipped with disposable needles (Honey Bee Insemination Service; http://www.honeybeeinsemination.com/equipment2.html). The needles were prepared from borosilicate capillary tubes $(0.8-1.10 \times 100 \mathrm{~mm})$ with a micropipette puller (Narishige Model PC-10, East Meadow, New York, USA). To investigate the role of dsRNA in honey bee antiviral defense, SINV-GFP was inoculated with different types of dsRNA ( $1 \mu \mathrm{g}$ each), including virus-specific dsRNA (sp-dsRNA, $928 \mathrm{bp}$ ), nonspecific dsRNA matching Drosophila C virus sequence (ns-dsRNA, 1,017 bp), or luciferase sequence (LUC dsRNA, 355 bp) (Supplementary Table S1). Bees were also co-injected with $1 \mu \mathrm{g}$ high molecular weight polyinosinic-polycytidylic acid (poly(I:C)), InvivoGen) or $1 \mu \mathrm{g}$ nucleoside triphosphates (NTP), the positive and negative controls. Mock-infection controls were also performed. Bees were collected at 6, 48, or 72 hours post-infection (hpi); a time frame that allowed for virus dissemination and infection, while maintaining optimal conditions for bees housed within the laboratory setting ${ }^{22}$.

dsRNA preparation. dsRNA was generated by in vitro transcription with T7 RNA polymerase ${ }^{109,112}$. T7 promoter containing dsDNA PCR-products were amplified using primers listed in Supplementary Table S1, with the following thermocycler program: pre-incubation of $95^{\circ} \mathrm{C}(5 \mathrm{~min}), 35 \mathrm{cycles}$ of $95^{\circ} \mathrm{C}(30 \mathrm{~s}), 60^{\circ} \mathrm{C}(30 \mathrm{~s})$, and $72^{\circ} \mathrm{C}(1 \mathrm{~min})$ followed by a final incubation at $72^{\circ} \mathrm{C}(5 \mathrm{~min})$. PCR products were templates for $\mathrm{T} 7$ polymerase transcription ( $100 \mu \mathrm{l}$ reactions: NTPs (each $7.5 \mathrm{mM}$ final), RNase OUT (40 units) (Invitrogen), buffer ( $400 \mathrm{mM}$ HEPES pH 7.5, $120 \mathrm{mM} \mathrm{MgCl}_{2}, 10 \mathrm{mM}$ Spermidine, $200 \mathrm{mM} \mathrm{DTT}$ ); reactions were carried out at $37^{\circ} \mathrm{C}$ overnight (8-10 hours). DNA was removed by incubating with RQ1 DNAse (1 unit; Promega) for 15 minutes at $37^{\circ} \mathrm{C}$. ssRNA products were ethanol precipitated, suspended in $200 \mu \mathrm{l}$ Rnase-free water, and annealed at $100^{\circ} \mathrm{C}$ for 5 minutes and then slowly cooled to room temperature. dsRNA products were purified by phenol:chloroform extraction and ethanol precipitation. Quality was assessed by agarose gel electrophoresis and spectrophotometry. The dsRNA quantity based on gel band intensity was assessed using Image ${ }^{113}$.

dsRNA-mediated gene knockdown. The expression of two candidate antiviral genes: dicer (XM_016917734.1) and a novel transcript with 91\% sequence identity with the A. cerana probable cyclin-dependent serine/threonine-protein kinase DDB_G0292550 (XM_017051141.1) was reduced by RNAi-mediated gene knockdown. In order to assess the effects of gene knockdown on virus abundance, bees were infected with SINV-GFP (using methods as above) and co-injected with $1 \mu \mathrm{g}$ of either gene-specific or nonspecific (DCV-specific) dsRNA (control) (Supplementary Table S1).

Honey bee RNA isolation and purification. TRizol reagent (Invitrogen) was added to individual bee thorax and abdomen homogenate and RNA was isolated following the manufacturer's instructions. Prior to gene expression analysis by RNASeq or qPCR, RNA was further purified using Qiagen RNAeasy columns including on-column DNase t reatment (Qiagen) to remove DNA from samples. RNA was quantified using a spectrophotometer.

Reverse transcription/cDNA synthesis. Reverse transcription reactions $(25 \mu \mathrm{l})$ were performed using $500 \mathrm{ng}$ of total RNA and random hexamer primers (500 ng) (IDT, Coralville, IA) incubated with Maloney murine leukemia virus (M-MLV) reverse transcriptase (Promega, Madison, WI) for 1 hour at $37^{\circ} \mathrm{C}$, according to the manufacturer's instructions.

Quantitative PCR (qPCR). Quantitative PCR was utilized to examine the relative abundance of virus and honey bee host gene expression in each sample using previously described methods that are in accordance with published guidelines ${ }^{114}$. All qPCR reactions were performed in triplicate using $2 \mu \mathrm{L}$ of cDNA as template. Each $20 \mu \mathrm{l}$ reaction was composed of cDNA template, 1X SYBR Green (Invitrogen), 1X Choice Taq Master Mix (Denville Scientific Inc.), $3 \mathrm{mM} \mathrm{MgCl}$, and forward and reverse primers (600 $\mathrm{nM}$ each). A CFX Connect Real Time instrument (BioRad) was utilized for qPCR, the thermo-profile for virus (e.g., SINV-GFP and BQCV) and A. mellifera rpl8 analyses consisted of a single pre-incubation $95^{\circ} \mathrm{C}(3 \mathrm{~min}), 40$ cycles of $95^{\circ} \mathrm{C}(5 \mathrm{~s}), 60^{\circ} \mathrm{C}(20 \mathrm{~s})$, $72{ }^{\circ} \mathrm{C}(30 \mathrm{~s})$, and a final elongation $72^{\circ} \mathrm{C}(4 \mathrm{~min})$ (Supplementary Table S1). Positive and negative controls, 
including the use of RNA templates from no RT enzyme cDNA reactions, were included for all qPCR analyses and exhibited the expected results.

To quantify the viral RNA (i.e., genome and transcript) abundance in each sample target SINV-GFP qPCR amplicons were cloned into the pGEM-T (Promega) vector, as described in Flenniken and Andino et al. ${ }^{22}$. Plasmid standards, containing $10^{9}$ to $10^{3}$ copies per reaction, were used as qPCR templates to assess primer efficiency and generate the standard curve used for viral genome copy quantification ${ }^{22}$. The qPCR primers for RNAseq validation were designed using Primer3Plus and with $60^{\circ} \mathrm{C}$ annealing temperatures ${ }^{115}$ (Supplementary Table S1). Melt point analysis and 2\% agarose gel electrophoresis ensured qPCR specificity ${ }^{116}$. Primer efficiencies were evaluated using qPCR assays of cDNA and plasmid dilution series, and calculated by plotting $\log _{10}$ of the concentration versus the crossing point threshold $(\mathrm{C}(\mathrm{t}))$ values and using the primer efficiency equation, $\left(10^{(1 / \text { Slope })-1)} \times 100\right)$ (Supplementary Table S16).

The $\Delta \Delta \mathrm{C}(\mathrm{t})$ method was used to calculate relative abundance of SINV-GFP in individual bees $(\mathrm{n}=10)$ because it was most accurate; the $\Delta \Delta \mathrm{C}(\mathrm{t})$ method ensures that results are not skewed by inadvertent differences in RNA reverse-transcription efficiencies and starting cDNA template abundance ${ }^{114,116,117}$. The $\Delta \mathrm{C}(\mathrm{t})$ for each sample was calculated by subtracting the Am rpl8 C(t) from the SINV-GFP C(t). The honey bee gene encoding ribosomal protein 8, Am rpl8, was selected as an appropriate housekeeping gene for qPCR because it has been utilized in several other studies ${ }^{118-121}$ and analysis of the RNASeq data presented herein confirmed that $r$ pl8 expression levels were similar in all sequenced libraries. The $\Delta \Delta \mathrm{C}(\mathrm{t})$ was calculated by subtracting the average virus-infected $\Delta \mathrm{C}(\mathrm{t})$ values from the $\Delta \mathrm{Ct}$ values for each treatment group. For host gene expression analyses and RNAseq validation, the percent gene expression for each gene of interest (GOI) was calculated using the following formula: $2^{-\Delta \Delta \mathrm{C}(\mathrm{t})} \times 100=\%$ gene expression, in which $\Delta \mathrm{C}(\mathrm{t})=\mathrm{GOI} \mathrm{C}(\mathrm{t})-\operatorname{rpl} l \mathrm{C}(\mathrm{t})$, and $\Delta \Delta \mathrm{C}(\mathrm{t})=$ sample $\Delta \mathrm{C}(\mathrm{t})-$ mock-infected control $\Delta \mathrm{C}(\mathrm{t})$. Based on previous work ${ }^{22,44}$, we hypothesized that bees $(\mathrm{n}=10)$ co-injected with dsRNA or poly(I:C) would have decreased relative virus abundance as compared to the virus-only treated group. To examine relative virus abundance between treatment groups (e.g., virus-infected bees and dsRNA or poly(I:C) co-treated bees) that had equal variance and normal distribution we performed one-tailed Student's t-tests. Analysis of honey bee host gene expression revealed unequal variance between treatments groups and thus Welch's t-tests were used to identify statistical differences in host gene expression.

RNAseq Library Preparation and Sequencing. Individual bee cDNA was screened for pre-existing infections via PCR for several honey bee pathogens (Supplementary Table S3) using the PCR thermocycler protocol: $95^{\circ} \mathrm{C}(5 \mathrm{~min}) ; 35 \mathrm{cycles}$ of $95^{\circ} \mathrm{C}(30 \mathrm{~s}), 57^{\circ} \mathrm{C}(30 \mathrm{~s})$, and $72^{\circ} \mathrm{C}(30 \mathrm{~s})$, followed by final elongation at $72^{\circ} \mathrm{C}$ for 4 minutes. If the sample was positive for a pathogen, the quantity was then assessed using qPCR. The RNA isolated from the abdomens of at least three representative bees with low $(<2,000 \mathrm{DWV}$ and/or BQCV virus genome copies versus $7 \times 10^{4}-7 \times 10^{6} \mathrm{SINV}$-GFP copies) to no pre-existing infections for each treatment group and time point were selected for transcriptome sequencing for a total of 47 individual bees (Supplementary Table S3).

Prior to RNASeq library preparation, RNA from each sample was further purified and DNase treated using Qiagen RNeasy columns. RNA quality was assessed using an Agilent 2200 Bioanalyzer and quantified via spectrophotometer. RNA was sent to the Roy J. Carver Biotechnology Center at the University of Illinois for library preparation (Illumina TruSeq Stranded RNA Sample Prep kit). Libraries were prepared and pooled by experimental time point and quantitated using an Illumina Library quantification kit (Kapa). Each pool was paired-end sequenced $(2 \times 100 \mathrm{nt})$ on a HiSeq. 2500 using a TruSeq SBS sequencing kit version 4 , yielding $\sim 12$ million reads per sample, corresponding to at least 9.7 fold coverage (Supplementary Table S2), which is in the range of coverage reported in other honey bee transcriptome studies ${ }^{24,25,122}$. Sequence data was deposited into the NCBI Sequence Read Archive under accession number SRP101337 and is linked with NCBI BioProject \#PRJNA377749.

FastQC and fastx-toolkit were used to remove low quality reads $(<\mathrm{Q} 30)$. Illumina adapters were trimmed with Trimmomatic ${ }^{123}$, and reads were aligned to the A. mellifera genome assembly 4.5 from NCBI with Tophat v2.0.14 5 ; on average, $\sim 77 \%$ of reads from each sample were mapped (Supplementary Table S2). The normalized number of Fragments Per Kilobase of transcript per Million mapped reads (FPKM) was determined using CuffDiff ${ }^{124,125}$ using the default classic FPKM normalization method and the default pooled dispersion model (Benjamini-Hochberg correction; significantly differentially expressed genes (DEGs) had q-value $\leq 0.05$ ). Venn diagrams were generated using Vennt ${ }^{126}$. To further investigate the function of the DEGs, representative protein sequences (the longest sequence if there were splice variants) of every known honey bee gene were blasted against the D. melanogaster protein database via reciprocal BLAST $+{ }^{127}$ to identify fruit fly orthologs and homologs of the honey bee genes because there is a greater amount of gene ontology information for D. melanogaster genes compared to Apis mellifera genes. The honey bee genome encodes approximately 15,000 genes of which 13,592 genes are mapped and provided in the Amel4.5 genome annotation ${ }^{128}$. We annotated 8,944 genes $(\sim 66 \%)$ as homologs (of which 7,006 were reciprocal best hits or orthologs) to genes encoded by the fruit fly D. melanogaster genome, which encodes $\sim 13,600$ genes ${ }^{128,129}$. Biological processes (BP) functional enrichment analysis was performed with DAVID $^{130}$. Gene ontology and biological processes (BP-FAT) enrichment analysis was performed with DAVID ${ }^{130}$.

Comparative analysis of DEGs in virus-infected bees. To identify the shared and unique DEGs in virus-infected bees, we compared our dataset to other studies that examined gene expression in virus-infected bees. Genes that were differentially expressed in SINV-GFP infected bees $72 \mathrm{hpi}$ were compared to other studies that examined gene expression in virus-infected bees: symptomatic IAPV-fed bees ${ }^{24}$; SBV and DWV-infected bees $^{25}$; adult honey bees naturally infected with IAPV ${ }^{9}$; and a common DEG list that was compiled from 19 gene expression data sets including Varroa destructor-parasitized and virus-infected bees ${ }^{92}$. We used NCBI Entrez Gene ID as a common identifier because DEG lists were generated using different technologies and versions of the Apis mellifera genome and transcriptome (Supplementary Table S14). DEGs were compared via Venn diagram 
analysis $^{131}$ (Supplementary Table S15). Pairwise comparisons between studies identified shared DEGs and the statistical significance of gene overlap was assessed using hypergeometric tests ${ }^{132}$ (Supplementary Table S15).

Identification of previously unrecognized honey bee transcript. RNASeq analysis determined that reads aligning to LOC725387 were more abundant in virus-infected bees. To identify the gene or genes encoded by these differentially expressed reads, the consensus nucleotide sequence was used to query the NCBI Nucleotide collection ( $\mathrm{nr} / \mathrm{nt}$ ) and A. mellifera databases using blastn ${ }^{133}$, Sanger sequencing was performed to verify transcript sequence and length, and the results were evaluated using Geneious ${ }^{134}$. Together, these analyses revealed that we identified a previously unrecognized transcript, $A$. mellifera probable cyclin-dependent serine/ threonine-protein kinase (MF116383, 5,158 nt), which is longer than the originally annotated A. mellifera probable serine/threonine-protein kinase clkA (LOC725387, XM_001121241.4, 1,403 nt).

In brief, we utilized LOC725387 RNASeq consensus sequence to query the NCBI Nucleotide nr/nt data base and identified an A. cerana transcript annotated as a probable cyclin-dependent serine/threonine-protein kinase DDB_G0292550 (LOC107994302,XM_017051141.1) as the top blastn result, which contained 95\% of the submitted sequence and shared $91 \%$ identity (E-value $=0,95 \%$ query coverage, $91 \%$ identity, $1-6 \%$ gaps); additional top blastn hits included $A$. dorsata GATA zinc finger domain-containing protein 14-like. When the LOC725387 RNASeq consensus sequence was used to query the A. mellifera database, the top blastn result only covered $24 \%$ of the query sequence (i.e., A. mellifera probable serine/threonine-protein kinase clkA, XM_001121241.4; $\mathrm{E}$-value $=0,24 \%$ query coverage, $99 \%$ identity, $0 \%$ gaps). To further characterize the LOC725387 transcript, we Sanger sequenced 5,027 nts $(\sim 2-3 \times$ coverage $)$ and obtained the most $5^{\prime}$ end of this transcript from RNASeq data (131 bp, $>2,000 \times$ coverage) (Supplementary Table S1 and Fig. S9). Together nucleotide and amino acid alignments indicate the RNAseq reads aligning to LOC725387 are most similar to a computationally predicted $A$. cerana cyclin-dependent serine/threonine-protein kinase DDB_G0292550 (Supplementary Fig. S9). Therefore, we refer to the gene identified herein as A. mellifera probable cyclin-dependent serine/threonine-protein kinase (Supplementary Fig. S9) and submitted the sequence of this transcript to NCBI (MF116383).

Data Availability. The majority of the data generated or analyzed during this study are included in this published article and its Supplementary Information files (available online), additional data is available from the corresponding author upon request, and sequence data may be accessed from the NCBI Sequence Read Archive (accession number SRP101337).

\section{References}

1. Gallai, N., Salles, J.-M., Settele, J. \& Vaissière, B. E. Economic valuation of the vulnerability of world agriculture confronted with pollinator decline. Ecol. Econ. 68, 810-821 (2009).

2. Calderone, N. W. Insect pollinated crops, insect pollinators and US agriculture: Trend analysis of aggregate data for the period 1992-2009. PLoS One 7, 24-28 (2012).

3. vanEngelsdorp, D. et al. Colony collapse disorder: a descriptive study. PLoS One 4, e6481 (2009).

4. Seitz, N. et al. A national survey of managed honey bee 2014-2015 annual colony losses in the USA. J. Apic. Res. 54, 292-304 (2015).

5. Chauzat, M.-P., Laurent, M., Ribiere-Chabert, M. \& Hendrikx, P. A pan-European epidemiological study on honeybee colony losses 2012-2014. Epilobee 2013, 44 (2016).

6. Cornman, R. S. et al. Pathogen webs in collapsing honey bee colonies. PLoS One 7, e43562 (2012)

7. Ravoet, J. et al. Comprehensive bee pathogen screening in Belgium reveals Crithidia mellificae as a new contributory factor to winter mortality. PLoS One 8, e72443 (2013).

8. Cox-Foster, D. L. et al. A metagenomic survey of microbes in honey bee colony collapse disorder. Science 318, 283-7 (2007).

9. Chen, Y. P. et al. Israeli acute paralysis virus: epidemiology, pathogenesis and implications for honey bee health. PLoS Pathog. 10, e1004261 (2014)

10. Daughenbaugh, K. F. et al. Honey Bee Infecting Lake Sinai Viruses. Viruses 7, 3285-3309 (2015).

11. van der Zee, R. et al. Managed honey bee colony losses in Canada, China, Europe, Israel and Turkey, for the winters of 2008-9 and 2009-10. J. Apic. Res. 51, 100-114 (2012).

12. Antúnez, K. et al. Seasonal Variation of Honeybee Pathogens and its Association with Pollen Diversity in Uruguay. Microb. Ecol. 70, 522-533 (2015).

13. Budge, G. E. et al. Pathogens as predictors of honey bee colony strength in England and Wales. PLoS One 10, 1-10 (2015).

14. Granberg, F. et al. Metagenomic detection of viral pathogens in Spanish honeybees: co-infection by Aphid Lethal Paralysis, Israel Acute Paralysis and Lake Sinai Viruses. PLoS One 8, e57459 (2013).

15. Nazzi, F. et al. Synergistic parasite-pathogen interactions mediated by host immunity can drive the collapse of honeybee colonies. PLoS Pathog. 8 (2012).

16. Porrini, C. et al. The status of honey bee health in Italy: Results from the nationwide bee monitoring network. PLoS One 11, 1-22 (2016).

17. Genersch, E. et al. The German bee monitoring project: a long term study to understand periodically high winter losses of honey bee colonies. Apidologie 41, 332-352 (2010).

18. Evans, J. D. \& Schwarz, R. S. Bees brought to their knees: microbes affecting honey bee health. Trends Microbiol. 19, 614-20 (2011).

19. Brutscher, L. M., McMenamin, A. J. \& Flenniken, M. L. The Buzz about Honey Bee Viruses. PLoS Pathog. 12, 1-7 (2016).

20. Chen, Y. \& Siede, R. Honey bee viruses. Adv. Virus Res. 70, 33-80 (2007).

21. Runckel, C. et al. Temporal analysis of the honey bee microbiome reveals four novel viruses and seasonal prevalence of known viruses, Nosema, and Crithidia. PLoS One 6, e20656 (2011).

22. Flenniken, M. L. \& Andino, R. Non-specific dsRNA-mediated antiviral response in the honey bee. PLoS One 8, e77263 (2013).

23. Bull, J. C. et al. A strong immune response in young adult honeybees masks their increased susceptibility to infection compared to older bees. PLoS Pathog. 8, e1003083 (2012).

24. Galbraith, Da, Yang, X., Niño, E. L., Yi, S. \& Grozinger, C. Parallel Epigenomic and Transcriptomic Responses to Viral Infection in Honey Bees (Apis mellifera). PLOS Pathog. 11, e1004713 (2015).

25. Ryabov, E. V., Fannon, J. M., Moore, J. D., Wood, G. R. \& Evans, D. J. The Iflaviruses Sacbrood virus and Deformed wing virus evoke different transcriptional responses in the honeybee which may facilitate their horizontal or vertical transmission. PeerJ 4, e1591 (2016). 
26. Ryabov, E. V. et al. A Virulent Strain of Deformed Wing Virus (DWV) of Honeybees (Apis mellifera) Prevails after Varroa destructor-Mediated, or In Vitro, Transmission. PLoS Pathog. 10, e1004230 (2014).

27. Bronkhorst, A. W. \& Van Rij, R. P. The long and short of antiviral defense: Small RNA-based immunity in insects. Curr. Opin. Virol. 7, 19-28 (2014)

28. Kingsolver, M. B. \& Hardy, R. W. Making connections in insect innate immunity. Proc. Natl. Acad. Sci. USA 109, 18639-40 (2012).

29. Kingsolver, M. B., Huang, Z. \& Hardy, R. W. Insect antiviral innate immunity: pathways, effectors, and connections. J. Mol. Biol. 425, 4921-36 (2013).

30. Galiana-Arnoux, D., Dostert, C., Schneemann, A., Hoffmann, Ja \& Imler, J.-L. Essential function in vivo for Dicer-2 in host defense against RNA viruses in drosophila. Nat. Immunol. 7, 590-7 (2006).

31. van Rij, R. P. et al. The RNA silencing endonuclease Argonaute 2 mediates specific antiviral immunity in Drosophila melanogaster. Genes Dev. 20, 2985-95 (2006).

32. Zambon, Ra, Vakharia, V. N. \& Wu, L. P. RNAi is an antiviral immune response against a dsRNA virus in Drosophila melanogaster. Cell. Microbiol. 8, 880-9 (2006).

33. Wang, X.-H. et al. RNA interference directs innate immunity against viruses in adult Drosophila. Science 312, $452-454$ (2006).

34. Myles, K. M., Wiley, M. R., Morazzani, E. M. \& Adelman, Z. N. Alphavirus-derived small RNAs modulate pathogenesis in disease vector mosquitoes. Proc. Natl. Acad. Sci. USA 105, 19938-43 (2008).

35. Brackney, D. E., Beane, J. E. \& Ebel, G. D. RNAi targeting of West Nile virus in mosquito midguts promotes virus diversification. PLoS Pathog. 5, el000502 (2009).

36. Maori, E. et al. IAPV, a bee-affecting virus associated with Colony Collapse Disorder can be silenced by dsRNA ingestion. Insect Mol. Biol. 18, 55-60 (2009).

37. Chejanovsky, N. et al. Characterization of viral siRNA populations in honey bee colony collapse disorder. Virology 454-455, 176-83 (2014)

38. Chen, Y. J. \& Evans, J. D. RNAi in Treating Honey Bee Diseases. Bee Cult. 140, 27-29 (2012).

39. Desai, S. D., Eu, Y.-J., Whyard, S. \& Currie, R. W. Reduction in deformed wing virus infection in larval and adult honey bees (Apis mellifera L.) by double-stranded RNA ingestion. Insect Mol. Biol. 21, 446-55 (2012).

40. Liu, X., Zhang, Y., Yan, X. \& Han, R. Prevention of Chinese Sacbrood Virus infection in Apis Cerana using RNA interference. Curr. Microbiol. 61, 422-428 (2010).

41. Hunter, W. et al. Large-scale field application of RNAi technology reducing Israeli acute paralysis virus disease in honey bees (Apis mellifera, hymenoptera: Apidae). PLoS Pathog. 6 (2010).

42. Piot, N., Snoeck, S., Vanlede, M., Smagghe, G. \& Meeus, I. The Effect of Oral Administration of dsRNA on Viral Replication and Mortality in Bombus terrestris. Viruses 7, 3172-3185 (2015).

43. Nunes, F. et al. Non-Target Effects of Green Fluorescent Protein (GFP)-Derived Double-Stranded RNA (dsRNA-GFP) Used in Honey Bee RNA Interference (RNAi) Assays. Insects 4, 90-103 (2013).

44. Niu, J., Meeus, I. \& Smagghe, G. Differential expression pattern of Vago in bumblebee (Bombus terrestris), induced by virulent and avirulent virus infections. Sci. Rep. 6, 34200 (2016).

45. Jarosch, A. \& Moritz, R. F. A. RNA interference in honeybees: off-target effects caused by dsRNA. Apidologie 43, 128-138 (2012).

46. Schoggins, J. W. \& Rice, C. M. Interferon-stimulated genes and their antiviral effector functions. Curr. Opin. Virol. 1, 519-525 (2011).

47. Paradkar, P. N., Trinidad, L., Voysey, R., Duchemin, J.-B. \& Walker, P. J. Secreted Vago restricts West Nile virus infection in Culex mosquito cells by activating the Jak-STAT pathway. Proc. Natl. Acad. Sci., doi:10.1073/pnas.1205231109 (2012).

48. Paradkar, P. N., Duchemin, J. B., Voysey, R. \& Walker, P. J. Dicer-2-Dependent Activation of Culex Vago Occurs via the TRAF-Rel2 Signaling Pathway. PLoS Negl. Trop. Dis. 8 (2014).

49. Deddouche, S. et al. The DExD/H-box helicase Dicer-2 mediates the induction of antiviral activity in drosophila. Nat. Immunol. 9 , $1425-1432$ (2008).

50. Heinemann, J. A., Agapito-Tenfen, S. Z. \& Carman, J. A. A comparative evaluation of the regulation of GM crops or products containing dsRNA and suggested improvements to risk assessments. Environ. Int. 55, 43-55 (2013).

51. Lundgren, J. G. \& Duan, J. J. RNAi-Based Insecticidal Crops. Bioscience 63, 657-665 (2013)

52. Kim, D. et al. TopHat2: accurate alignment of transcriptomes in the presence of insertions, deletions and gene fusions. Genome Biol. 14, R36 (2013)

53. Marringa, W. J., Krueger, M. J., Burritt, N. L. \& Burritt, J. B. Honey bee hemocyte profiling by flow cytometry. PLoS One 9, e108486 (2014).

54. Sagan, S. M. \& Sarnow, P. Molecular biology. RNAi, Antiviral after all. Science 342, 207-8 (2013)

55. Saleh, M.-C. et al. The endocytic pathway mediates cell entry of dsRNA to induce RNAi silencing. Nat. Cell Biol. 8, 793-802 (2006).

56. Brutscher, L. M., Daughenbaugh, K. F. \& Flenniken, M. L. Antiviral defense mechanisms in honey bees. Curr. Opin. Insect Sci. 2, $1-12(2015)$

57. Costa, A., Jan, E., Sarnow, P. \& Schneider, D. The Imd pathway is involved in antiviral immune responses in Drosophila. PLoS One 4, e7436 (2009).

58. Avadhanula, V., Weasner, B. P., Hardy, G. G., Kumar, J. P. \& Hardy, R. W. A novel system for the launch of alphavirus RNA synthesis reveals a role for the Imd pathway in arthropod antiviral response. PLoS Pathog. 5, e1000582 (2009).

59. Mandrioli, M., Monti, M. \& Tedeschi, R. Presence and conservation of the immunoglobulin superfamily in insects: current perspective and future challenges. Invertebr. Surviv. J. 12, 188-194 (2015).

60. Hirai, M., Terenius, O., Li, W. \& Faye, I. Baculovirus and dsRNA induce Hemolin, but no antibacterial activity, in Antheraea pernyi. Insect Mol. Biol. 13, 399-405 (2004).

61. Nainu, F., Tanaka, Y., Shiratsuchi, A. \& Nakanishi, Y. Protection of Insects against Viral Infection by Apoptosis-Dependent Phagocytosis. J. Immunol. 195, 5696-706 (2015).

62. Ulvila, J. et al. Double-stranded RNA is internalized by scavenger receptor-mediated endocytosis in Drosophila S2 cells. J. Biol. Chem. 281, 14370-14375 (2006).

63. Yamauchi, Y. \& Helenius, A. Virus entry at a glance. J. Cell Sci. 126, 1289-1295 (2013).

64. Leung, J. Y. S., Ng, M. M. L. \& Chu, J. J. H. Replication of alphaviruses: A review on the entry process of alphaviruses into cells. $A d v$. Virol. 2011 (2011).

65. Wang, K.-S., Kuhn, R. J., Strauss, E. G., Ou, S. \& Strauss, J. H. High-Affinity Laminin Receptor Is a Receptor for Sindbis Virus in Mammalian Cells. J. Virol. 66, 4992-5001 (1992).

66. Bou Aoun, R. et al. Analysis of thioester-containing proteins during the innate immune response of Drosophila melanogaster. J. Innate Immun. 3, 52-64 (2011).

67. Cheng, G. et al. An in vivo transfection approach elucidates a role for Aedes aegypti Thioester-Containing proteins in flaviviral infection. PLoS One 6, 1-7 (2011).

68. Lighthouse, D. V., Buszczak, M. \& Spradling, A. C. New components of the Drosophila fusome suggest it plays novel roles in signaling and transport. Dev. Biol. 317, 59-71 (2008)

69. Shaw, A. S. \& Filbert, E. L. Scaffold proteins and immune-cell signalling. Nat. Rev. Immunol. 9, 47-56 (2009).

70. Aggarwal, K. \& Silverman, N. Positive and negative regulation of the Drosophila immune response. BMB Rep. 41, 267-277 (2008). 
71. Ragab, A. et al. Drosophila Ras/MAPK signalling regulates innate immune responses in immune and intestinal stem cells. EMBO J. 30, 1123-36 (2011).

72. Kleino, A. et al. Inhibitor of apoptosis 2 and TAK1-binding protein are components of the Drosophila Imd pathway. EMBO J. 24, 3423-34 (2005)

73. Vlisidou, I. \& Wood, W. Drosophila blood cells and their role in immune responses. FEBS J. 282, 1368-1382 (2015).

74. Clevers, H. Wnt/ $\beta$-Catenin Signaling in Development and Disease. Cell 127, 469-480 (2006).

75. Evans, J. D. et al. Immune pathways and defence mechanisms in honey bees Apis mellifera. Insect Mol. Biol. 15, 645-56 (2006).

76. Li, W.-F., Ma, G.-X. \& Zhou, X.-X. Apidaecin-type peptides: Biodiversity, structure-function relationships and mode of action. Peptides 27, 2350-2359 (2006).

77. Erler, S., Popp, M. \& Lattorff, H. M. G. Dynamics of immune system gene expression upon bacterial challenge and wounding in a social insect (Bombus terrestris). PLoS One 6 (2011).

78. Huang, Z., Kingsolver, M. B., Avadhanula, V. \& Hardy, R. W. An Antiviral Role for Antimicrobial Peptides during the Arthropod Response to Alphavirus Replication. J. Virol. 87, 4272-4280 (2013).

79. Schlüns, H. \& Crozier, R. H. Relish regulates expression of antimicrobial peptide genes in the honeybee, Apis mellifera, shown by RNA interference. Insect Mol. Biol. 16, 753-9 (2007).

80. Haslbeck, M. \& Vierling, E. A first line of stress defense: Small heat shock proteins and their function in protein homeostasis. J. Mol. Biol. 427, 1537-1548 (2015).

81. Merkling, S. H. et al. The heat shock response restricts virus infection in Drosophila. Sci. Rep. 5, 12758 (2015).

82. Miyoshi, T., Takeuchi, A., Siomi, H. \& Siomi, M. C. A direct role for Hsp90 in pre-RISC formation in Drosophila. Nat. Struct. Mol. Biol. 17, 1024-1026 (2010).

83. Pare, J. M. et al. Hsp90 Regulates the Function of Argonaute 2 and Its Recruitment to Stress Granules and P-Bodies. Mol. Biol. Cell 20, 3273-3284 (2009).

84. Kampmueller, K. M. \& Miller, D. J. The cellular chaperone heat shock protein 90 facilitates Flock House virus RNA replication in Drosophila cells. J. Virol. 79, 6827-6837 (2005).

85. Tsou, Y.-L. et al. Heat shock protein 90: role in enterovirus 71 entry and assembly and potential target for therapy. PLoS One 8 , e77133 (2013)

86. Brutscher, L. M. \& Flenniken, M. L. RNAi and Antiviral Defense in the Honey Bee. J. Immunol. Res. 2015, 1-10 (2015).

87. Mitoma, H. et al. The DHX33 RNA Helicase Senses Cytosolic RNA and Activates the NLRP3 Inflammasome. Immunity 39, $123-135(2013)$

88. Boncristiani, H. F. et al. In Vitro Infection of Pupae with Israeli Acute Paralysis Virus Suggests Disturbance of Transcriptional Homeostasis in Honey Bees (Apis mellifera). PLoS One 8 (2013).

89. Di Prisco, G. et al. A mutualistic symbiosis between a parasitic mite and a pathogenic virus undermines honey bee immunity and health. Proc. Natl. Acad. Sci. 113, 201523515 (2016).

90. Steinmann, N., Corona, M., Neumann, P. \& Dainat, B. Overwintering Is Associated with Reduced Expression of Immune Genes and Higher Susceptibility to Virus Infection in Honey Bees. PLoS One 10, e0129956 (2015).

91. Li, W. et al. Silencing honey bee (Apis mellifera) naked cuticle (nkd) improves host immune function and reduces Nosema ceranae infections. Appl. Environ. Microbiol., doi:10.1128/AEM.02105-16 (2016).

92. Doublet, V. et al. Unity in defence: honeybee workers exhibit conserved molecular responses to diverse pathogens. BMC Genomics 18, 207 (2017)

93. Kurzik-Dumke, U. \& Lohmann, E. Sequence of the new Drosophila melanogaster small heat-shock-related gene, lethal(2) essential for life [1(2) efl], at locus 59F4,5. Gene 154, 171-175 (1995).

94. Nazzi, F., Annoscia, D., Caprio, E., Di Prisco, G. \& Pennacchio, F. Honeybee immunity and colony losses. Entomologia 2 (2014).

95. Tantin, D. Oct transcription factors in development and stem cells: insights and mechanisms. Development 140, 2857-66 (2013).

96. Cremer, S., Armitage, S. A. O. \& Schmid-Hempel, P. Social Immunity. Curr. Biol. 17, 693-702 (2007).

97. McMenamin, A. J., Brutscher, L. M., Glenny, W. \& Flenniken, M. L. Abiotic and biotic factors affecting the replication and pathogenicity of bee viruses. Curr. Opin. Insect Sci. 16, 14-21 (2016).

98. McMahon, D. P. et al. Elevated virulence of an emerging viral genotype as a driver of honeybee loss. Proc. Biol. Sci. 283, 443-449 (2016).

99. DeGrandi-Hoffman, G. \& Chen, Y. Nutrition, immunity and viral infections in honey bees. Curr. Opin. Insect Sci. 10, 170-176 (2015).

100. Tarpy, D. R. Genetic diversity within honeybee colonies prevents severe infections and promotes colony growth. Proc. R. Soc. London. Ser. B Biol. Sci. 270, 99-103 (2003).

101. Tarpy, D. R. \& Seeley, T. D. Lower disease infections in honeybee (Apis mellifera) colonies headed by polyandrous vs monandrous queens. Naturwissenschaften 93, 195-199 (2006).

102. Lopes, L. Q. S., Quatrin, P. M., De Souza, M. E. \& De Almeida Vaucher, R. \& Vienna Santos, C. R. S. Fungal Infections In Honey Bees. Fungal Genomics Biol. 5, 1-4 (2015).

103. Villa, J. D., Bourgeois, A. L. \& Danka, R. G. Negative evidence for effects of genetic origin of bees on Nosema ceranae, positive evidence for effects of Nosema ceranae on bees. Apidologie 44, 511-518 (2013).

104. Simone-Finstrom, M., Walz, M. \& Tarpy, D. R. Genetic diversity confers colony-level benefits due to individual immunity. Biol. Lett. 12, 20151007 (2016).

105. Kuster, R. D., Boncristiani, H. F. \& Rueppell, O. Immunogene and viral transcript dynamics during parasitic Varroa destructor mite infection of developing honey bee (Apis mellifera) pupae. J. Exp. Biol. 217, 1710-8 (2014).

106. Natsopoulou, M. E., Mcmahon, D. P., Doublet, V., Bryden, J. \& Paxton, R. J. Interspecific competition in honeybee intracellular gut parasites is asymmetric and favours the spread of an emerging infectious disease. Proc. Biol. Sci. 282, 20141896 (2015).

107. Carrillo-Tripp, J. et al. In vivo and in vitro infection dynamics of honey bee viruses. Sci. Rep. 6, 22265 (2016).

108. Evans, J. D., Chen, Y. P., Prisco, G., Pettis, J. \& Williams, V. Bee cups: single-use cages for honey bee experiments. 48, 300-302 (2009).

109. Saleh, M., Tassetto, M., Rij, R., Van \& Goic, B. Antiviral immunity in Drosophila requires systemic RNA interference spread. Nature 458, 346-350 (2009)

110. Nayak, A. et al. Cricket paralysis virus antagonizes Argonaute 2 to modulate antiviral defense in Drosophila. Nat. Struct. Mol. Biol. 17, 547-554 (2010).

111. Venticinque, L. \& Meruelo, D. Sindbis viral vector induced apoptosis requires translational inhibition and signaling through Mcl-1 and Bak. Mol. Cancer 9, 37 (2010).

112. Amdam, G. V., Simões, Z. L. P., Guidugli, K. R., Norberg, K. \& Omholt, S. W. Disruption of vitellogenin gene function in adult honeybees by intra- abdominal injection of double-stranded RNA. BMC Biotechnol. 8, 1-8 (2003).

113. Schneider, Ca, Rasband, W. S. \& Eliceiri, K. W. NIH Image to ImageJ: 25 years of image analysis. Nat. Methods 9, 671-675 (2012).

114. Huggett, J. F. et al. The digital MIQE guidelines: Minimum information for publication of quantitative digital PCR experiments. Clin. Chem. 59, 892-902 (2013).

115. Untergasser, A. et al. Primer3-new capabilities and interfaces. Nucleic Acids Res. 40, 1-12 (2012).

116. Ginzinger, D. G. Gene quantification using real-time quantitative PCR: an emerging technology hits the mainstream. Exp. Hematol. 30, 503-12 (2002). 
117. Scientific, T. Real-time PCR. Scientific, ThermoFisher (2016).

118. Evans, J. D. Beepath: an ordered quantitative-PCR array for exploring honey bee immunity and disease. J. Invertebr. Pathol. 93, 135-9 (2006)

119. Hou, C. S., Li, B. B., Deng, S. \& Diao, Q. Y. Effects of Varroa destructor on temperature and humidity conditions and expression of energy metabolism genes in infested honeybee colonies. Genet. Mol. Res. 15 (2016).

120. Hou, C., Rivkin, H., Slabezki, Y. \& Chejanovsky, N. Dynamics of the presence of israeli acute paralysis virus in honey bee colonies with colony collapse disorder. Viruses 6, 2012-27 (2014).

121. De Smet, L. et al. BeeDoctor, a versatile MLPA-based diagnostic tool for screening bee viruses. PLoS One 7, e47953 (2012).

122. Atallah, J., Plachetzki, D. C., Jasper, W. C. \& Johnson, B. R. The utility of shallow RNA-Seq for documenting differential gene expression in genes with high and low levels of expression. PLoS One 8, 1-11 (2013).

123. Trimmomatic. a flexible trimmer for Illumina sequence data. Bioinformatics 30, 2114 (2014).

124. Trapnell, C. et al. Differential gene and transcript expression analysis of RNA-seq experiments with TopHat and Cufflinks. Nat. Protoc. 7, 562-78 (2012)

125. Trapnell, C. et al. Differential analysis of gene regulation at transcript resolution with RNA-seq. Nat. Biotechnol. 31, 46-53 (2013).

126. Powell, D. R. Vennt: Dynamic Venn tool for differential gene expression. (2014). Available at: http://drpowell.github.io/vennt/. (Accessed: 1st August 2015).

127. Camacho, C. et al. BLAST plus: architecture and applications. BMC Bioinformatics 10, 1 (2009).

128. Elsik, C. G. et al. Finding the missing honey bee genes: lessons learned from a genome upgrade. BMC Genomics 15, 86 (2014).

129. Weinstock, G. M. et al. Insights into social insects from the genome of the honeybee Apis mellifera. Nature 443, 931-949 (2006).

130. Dennis, G. Jr. et al. DAVID: Database for Annotation, Visualization, and Integrated Discovery. Genome Biol. 4, P3 (2003).

131. Venn. Available at: http://bioinformatics.psb.ugent.be/webtools/Venn/. (Accessed: 3rd January 2017).

132. Alaux, C. et al. Honey bee aggression supports a link between gene regulation and behavioral evolution. Proc. Natl. Acad. Sci. 106, 15400-15405 (2009).

133. Altschul, S. F., Gish, W., Miller, W., Myers, E. W. \& Lipman, D. J. Basic local alignment search tool. J. Mol. Biol. 215, 403-10 (1990).

134. Kearse, M. et al. Geneious Basic: An integrated and extendable desktop software platform for the organization and analysis of sequence data. Bioinformatics 28, 1647-1649 (2012).

\section{Acknowledgements}

The Flenniken Laboratory is supported by the National Sciences Foundation CAREER Program, the United States Department of Agriculture National Institute of Food and Agriculture, Agriculture and Food Research Initiative (USDA-NIFA-AFRI) Program, Montana Department of Agriculture Specialty Crop Block Grant Program, the National Institutes of Health IDeA Program COBRE grant GM110732, National Science Foundation EPSCoR NSF-IIA-1443108, Hatch Multistate Funding (NC-1173), Project Apis m., the Montana State Beekeepers Association, Montana State University, and the Montana State University Agricultural Experiment Station. Laura M. Brutscher was supported by the Project Apis m.-Costco Honey Bee Biology Fellowship. We would like to thank members of the Flenniken laboratory (Alex McMenamin, William Glenny, and Vanessa Orcutt) for reviewing this manuscript prior to publication and Dr. Charles Carey for bioinformatics assistance.

\section{Author Contributions}

M.L.F. and L.M.B. conceived and designed the experiments; L.M.B., K.F.D., and M.L.F. conducted the experiments; L.M.B. and M.L.F. analyzed the results; L.M.B. and M.L.F. wrote the manuscript and all authors reviewed the manuscript.

\section{Additional Information}

Supplementary information accompanies this paper at doi:10.1038/s41598-017-06623-Z

Competing Interests: The authors declare that they have no competing interests.

Publisher's note: Springer Nature remains neutral with regard to jurisdictional claims in published maps and institutional affiliations.

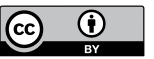

Open Access This article is licensed under a Creative Commons Attribution 4.0 International License, which permits use, sharing, adaptation, distribution and reproduction in any medium or format, as long as you give appropriate credit to the original author(s) and the source, provide a link to the Creative Commons license, and indicate if changes were made. The images or other third party material in this article are included in the article's Creative Commons license, unless indicated otherwise in a credit line to the material. If material is not included in the article's Creative Commons license and your intended use is not permitted by statutory regulation or exceeds the permitted use, you will need to obtain permission directly from the copyright holder. To view a copy of this license, visit http://creativecommons.org/licenses/by/4.0/.

(c) The Author(s) 2017 